\title{
Probing the strange content of the proton with charm production in charged current at $\mathrm{LHeC}$
}

\author{
XFITTER Developers' team: Hamed Abdolmaleki ${ }^{1}$, Valerio Bertone ${ }^{2}$, Daniel Britzger ${ }^{3}$, Stefano Camarda ${ }^{4}$, \\ Amanda Cooper-Sarkar ${ }^{5}$, Achim Geiser ${ }^{6}$, Francesco Giuli ${ }^{7}$, Alexander Glazov ${ }^{6}$, Agnieszka Luszczak ${ }^{8}$, \\ Ivan Novikov $^{9}$, Fred Olness ${ }^{10, a}$, Andrey Sapronov ${ }^{9}$, Oleksandr Zenaiev $^{11}$ \\ ${ }^{1}$ Faculty of Physics, Semnan University, 35131-19111 Semnan, Iran \\ 2 Dipartimento di Fisica, Università di Pavia and INFN, Sezione di Pavia Via Bassi 6, 27100 Pavia, Italy \\ ${ }^{3}$ Max-Planck-Institut für Physik, Föhringer Ring 6, 80805 Munich, Germany \\ ${ }^{4}$ CERN, 1211 Geneva 23, Switzerland \\ ${ }^{5}$ Particle Physics, University of Oxford, Denys Wilkinson Bdg, Keble Road, Oxford OX1 3RH, UK \\ ${ }^{6}$ Deutsches Elektronen-Synchrotron (DESY), Notkestrasse 85, 22607 Hamburg, Germany \\ ${ }^{7}$ University of Rome Tor Vergata and INFN, Sezione di Roma 2, Via della Ricerca Scientifica 1, 00133 Rome, Italy \\ ${ }^{8}$ T. Kosciuszko Cracow University of Technology, 30-084 Cracow, Poland \\ ${ }^{9}$ Joint Institute for Nuclear Research, Joliot-Curie 6, Dubna, Moscow Region 141980, Russia \\ ${ }^{10}$ SMU Physics, Box 0175, Dallas, TX 75275-0175, USA \\ ${ }^{11}$ Hamburg University, II. Institute for Theoretical Physics, Luruper Chaussee 149, 22761 Hamburg, Germany
}

Received: 2 July 2019 / Accepted: 1 October 2019 / Published online: 22 October 2019

(C) The Author(s) 2019

\begin{abstract}
We study charm production in charged-current deep-inelastic scattering (DIS) using the XFITTER framework. Recent results from the LHC have focused renewed attention on the determination of the strange-quark parton distribution function (PDF), and the DIS charm process provides important complementary constraints on this quantity. We examine the current PDF uncertainty and use LHeC pseudodata to estimate the potential improvement from this proposed facility. As XFITTER implements both fixed-flavorand variable-flavor-number schemes, we can compare the impact of these different theoretical choices; this highlights some interesting aspects of multi-scale calculations. We find that the high-statistics $\mathrm{LHeC}$ data covering a wide kinematic range could substantially reduce the strange PDF uncertainty.
\end{abstract}

\section{Contents}

1 Introduction . . . . . . . . . . . 1

2 Theoretical predictions for $\mathrm{CC}$ charm production at the $\mathrm{LHeC} \ldots \ldots \ldots$. . . . . . . . . . . . . 2

2.1 The heavy-flavor schemes . . . . . . . . . . . 3

2.2 The reduced cross section . . . . . . . . . . . 3

2.3 xFITTER implementation . . . . . . . . . . 4

3 Comparison of theoretical predictions . . . . . . . 4

a e-mail: olness@smu.edu
3.1 Comparison of theoretical predictions in the FFNS A and FONLL-B schemes . . . . . . . 4

3.2 Additional comparisons . . . . . . . . . . . 5

3.3 Contributions from different partonic subprocesses . 8

4 PDF constraints from charm CC pseudodata ... . 9

4.1 The CC charm pseudodata . . . . . . . . . 10

4.2 The profiled PDFs . . . . . . . . . . . . 10

5 Discussion and summary . . . . . . . . . . 12

Appendix A: $F_{2}^{c}$ Beyond leading-order . . . . . . 14

References . . . . . . . . . . . . . . 18

\section{Introduction}

The deep-inelastic-scattering (DIS) experiments traditionally have provided important tests of perturbative QCD (pQCD) and are essential to precisely determine the parton distribution functions (PDFs) of the nucleon. In addition to the numerous dedicated fixed-target DIS experiments that have been performed so far, the HERA accelerator used colliding beams of leptons (electrons and positrons) and protons to investigate the nucleon structure. The broad kinematic coverage of the HERA charge-current (CC) and neutral-current (NC) DIS data in terms of the negative virtuality $Q^{2}$ of the exchanged vector boson and the Bjorken variable $x_{\mathrm{Bj}}$ is such that these data have significant impact on the determinations of the PDFs [1-5]. 
In the Standard Model (SM), the charm quark plays an important role in the investigation of the nucleon structure [69]. In the $\mathrm{NC}$ case, the photon-gluon fusion process for charm production was calculated at $\mathscr{O}\left(\alpha_{s}^{2}\right)$ with the full heavy-quark mass dependence included in the DIS hard cross sections $[10,11]$. The heavy-quark mass effects in the CC process have been calculated to $\mathscr{O}\left(\alpha_{s}\right)$ in Refs. [12-16], and the recent work of Ref. [17] provides results up to $\mathscr{O}\left(\alpha_{s}^{2}\right)$. The large- $Q^{2}$ contributions of heavy flavors to the $x F_{3}$ structure function had already been computed in Ref. [18]. In many of the posited models which extend the SM, the coupling to "new physics" is proportional to the particle mass; hence, the heavy quarks will have an enhanced coupling and provide an optimal testing ground for these searches.

Heavy quarks also play a critical role in helping us fully characterize the SM, and the charm quark is especially useful in this respect as it can provide us direct access to the strange-sea quark distribution. The strange sea has been extensively investigated in a number of experiments including the associated production of a $W$ boson with a charm-jet final state, which (at LO) arises from strangegluon initial states [19-26]. Additionally, charm production in neutrino/antineutrino-nucleon DIS has been studied by a number of experiments including: CCFR [27], NuTeV [28], CHORUS [29], CDHSW [30] and NOMAD [31]. With a sign-selected beam $(v / \bar{v})$, these experiments can separately probe the strange $s(x)$ and anti-strange $\bar{s}(x)$ distributions. While the neutrino DIS experiments provide detailed information on the shape of the strange distribution, the normalization is a challenge, as that is tied to the beam flux. Separately, the HERMES collaboration used charged-lepton DIS production of charged kaons to provide a complementary extraction of $s(x)+\bar{s}(x)$ at LO [32]. Recently, charm production in CC DIS was measured for the first time in $e^{ \pm} p$ collisions by ZEUS [33].

Additionally, charm production mediated by electroweak gauge boson at hadron colliders provides important information on the strange- and charm-quark distributions, and is complementary to the DIS final-state charm-quark experiments [34]. The Tevatron measured the charm-quark cross section in association with a $W$ boson at CDF $[19,35,36]$ and D0 [20], but these results were limited by low statistics.

In lieu of significant experimental constraints, many global QCD analyses tie the strange distribution to the lightsea quarks via the relation $s=\bar{s}=r_{s} \bar{d}$. While in principle $r_{s}$ depends on both $x_{\mathrm{Bj}}$ and $Q^{2}$, it is often set to a fixed value $[37,38]$.

Using inclusive leptonic decays of $W$ and $Z$ bosons, the ATLAS experiment has obtained a value of $r_{s}=1.19 \pm$ 0.16 at $x=0.023$ and $Q_{0}^{2}=1.9 \mathrm{GeV}^{2}$ [39]. Additionally, using the cross section ratio for $W^{ \pm}+c$ final states they also find a comparably large value for $r_{s}$ [23]. In contrast, CMS results generally prefer lower $r_{s}$ values [22,24]. However, a recent analysis using both ATLAS and CMS data suggests that the LHC data support unsuppressed strangeness in the proton. While the result is dominated by ATLAS, this is not in contradiction with the CMS data $[22,23,39,40]$.

Looking to the future, it is clearly important to reduce the uncertainty of the strange-quark PDF as we strive to make increasingly precise tests of the SM and search for what might lie beyond. The proposed Large Hadron Electron Collider ( $\mathrm{LHeC}$ ) program has the ability to provide high statistics measurements of electrons on both protons and nuclei across a broad kinematic range to address many of these outstanding questions.

In this investigation, we make use of the XFITTER tools [41] (version 2.0.0) to study the present constraints on the strange-quark PDFs, and then use LHeC pseudodata [42] to infer how these might improve. Furthermore, as XFITTER implements both fixed-flavor- and variable-flavornumber schemes, we can examine the impact of these different theoretical choices.

This paper is organized as follows. In Sect. 2 we outline the theoretical details of the different heavy-flavor schemes. In Sect. 3 we compare the theoretical predictions of the different schemes across the kinematic range, and examine the individual partonic contributions. In Sect. 4 we study the impact of the $\mathrm{LHeC}$ pseudodata on the PDFs using a profiling technique. In Sect. 5 we provide some discussion and summarize the results. Finally, in Appendix A we discuss some of the more subtle theoretical issues that we encounter at higher orders.

\section{Theoretical predictions for $\mathrm{CC}$ charm production at the $\mathrm{LHeC}$}

The proposed Large Hadron Electron Collider (LHeC) [42] would collide a newly built electron beam with the LHC hadron beam at a center of mass energy of $\sqrt{s}=\sqrt{4 E_{e} E_{p}}$; thus the $7 \mathrm{TeV}$ proton beam on a $60 \mathrm{GeV}$ electron beam provide $\sqrt{s} \sim 1.3 \mathrm{TeV}$. Compared to HERA, the LHeC extends the covered kinematic range by an order of magnitude in both $x_{\mathrm{Bj}}$ and $Q^{2}$ with a nominal design luminosity of $10^{33} \mathrm{~cm}^{-2} \mathrm{~s}^{-1}$.

Theoretical predictions are calculated for electroweak charged-current (CC) charm production in ep collisions at the $\mathrm{LHeC}$ at centre-of-mass energy $\sqrt{s}=1.3 \mathrm{TeV}$, using a variety of heavy-flavor schemes. The predictions are provided for unpolarized beams in the kinematic range $100<Q^{2}<$ $100000 \mathrm{GeV}^{2}, 0.0001<x_{\mathrm{Bj}}<0.25$. They are calculated as reduced cross sections at different $Q^{2}, x_{\mathrm{Bj}}$ and inelasticity (y) points. The covered $y$ range is $0.0024<y<0.76$.

Experimentally, however, not charm quarks but charmed hadrons (or rather their decay products) are registered in the detectors. Therefore, extrapolation to the inclusive charm- 
production cross section has to be carried out in a modeldependent way. Furthermore, CC production of charm quarks in the final state can happen via both electroweak and QCD processes. The former leads to an odd number of charm quarks in the final state with the $W$ boson having the same electric charge as the sum of the electric charges of finalstate charm quarks, while the latter creates an even number of charm quarks with total electric charge equal to zero. If the electric charge of the tagged charm quark can be accessed experimentally (e.g. when reconstructing $D$ mesons), the QCD contribution can be subtracted by taking the difference of the yields in the events with odd and even numbers of charm quarks, otherwise the QCD contribution can be estimated only in a model-dependent way.

The CC charm process directly depends on the CKM matrix [43]. Here, the CKM matrix elements $V_{c d}$ and $V_{c s}$ are particularly relevant and we use the values $V_{c d}=0.2252$ and $V_{c s}=0.9734$. Three different heavy-flavor schemes are employed, all including a full treatment of charm-mass effects up to NLO, i.e. $\mathscr{O}\left(\alpha_{s}\right)$; in the following we describe them in detail for the particular application to $\mathrm{CC}$ electronproton reactions.

\subsection{The heavy-flavor schemes}

The standard " $A$ " variant of the fixed-flavor number scheme (FFNS), which we identify as FFNS A, uses three light flavors in both PDFs and $\alpha_{s}$ evolution for all scales, while heavy flavors (here, charm) are produced exclusively in the matrixelement part of the calculation. This scheme has been used for the PDF determinations and cross section predictions of the ABM(P) group [4,44-46], as well as in the FF3A variant of the HERAPDF analysis [2], and implemented in XFITTER through the OPENQCDRAD package [47].

Next, the "B" variant of the FFNS (FFNS B), known as the "mixed" or "hybrid" scheme [6] is also used. In this scheme, the number of active flavors is still fixed to three in the PDFs, relying exclusively on $\mathscr{O}\left(\alpha_{s}\right)$ fully massive matrix elements for charm production, while the number of flavors is allowed to vary in the virtual corrections of the $\alpha_{s}$ evolution. Corrections to the $\alpha_{s}$ evolution involving heavy-flavor loops are thus included and resummed to all orders, while no resummation is applied to other higher order corrections. This procedure will catch a fraction of the "large logs" which might spoil the fixed-flavor scheme convergence at very high scales, and is possible since the masses of the charm and beauty quarks provide natural cutoffs for infrared and collinear divergences. This scheme was used in the HERAPDF FF3B variant [2] and in applications of the HVQDIS program [6]. In general, the transition from the FFNS A to the FFNS B requires a readjustment of the treatment of matrix elements involving heavy-flavor loops. In the specific case of CC production, no such loops occur up to NLO (at NNLO they do), so that the same matrix elements can be used for both schemes; thus the only difference is in the $\alpha_{s}$ evolution.

Finally, for the variable-flavor-number scheme (VFNS) we use the "B" variant of the fixed-order-next-to-leading-log scheme (FONLL-B) [48] which combines the NLO $\mathscr{O}\left(\alpha_{S}\right)$ massive matrix elements of the FFNS with the $\mathscr{O}\left(\alpha_{s}\right)$ massless results of the zero-mass variable-flavor-number scheme (ZM-VFNS), allowing the number of active flavors to vary with scale, and all-order next-to-leading log resummation of (massless) terms beyond NLO. It thus explicitly includes charm and beauty both in the PDFs and in the evolution of the strong coupling constant. Whenever terms would be double-counted in the merging of the two schemes, the massless terms are eliminated in favour of the massive ones. The FONLL scheme is commonly used by the NNPDF group [5] and implemented in XFITTER through the APFEL package [49].

In summary, the schemes used are:

- FFNS A: a NLO FFNS with $n_{f}=3$ at all scales, used with the ABMP16 [46] or HERAPDF2.0 FF3A [2] NLO PDF sets.

- FFNS B: a NLO FFNS with $n_{f}=3$ for the PDFs and variable $n_{f}$ for $\alpha_{s}$, used with the HERAPDF2.0 FF3B [2] NLO PDF set.

- FONLL-B : a VFNS used with the NNPDF3.1 NLO PDF set [5].

The PDF sets are available via the LHAPDF interface (version 6.1.5) [50]. Note that we use the PDFs directly from the LHAPDF tables without any additional fitting, and we use the default values for $\alpha_{s}\left(M_{Z}\right)$ and the quark masses $m_{c, b}$. We've chosen this collection of PDFs as they are consistently extracted in the appropriate VFNS/FFNS schemes. Comparing the PDFs at the inital evolution scale, we find a typical variation of $\lesssim 10 \%$ for the quarks, and a bit larger for the gluon (which enters at NLO). These differences will lead to a small shift in the ratio plots, but will not affect the general features which are the focus of this paper, c.f., Fig. 2.

\subsection{The reduced cross section}

The reduced CC charm-production cross sections can be expressed as a linear combination of structure functions:

$\sigma_{\text {charm,CC }}^{ \pm}=\frac{1}{2}\left(Y_{+} F_{2}^{ \pm} \mp Y_{-} x F_{3}^{ \pm}-y^{2} F_{L}^{ \pm}\right)$

with

$Y_{ \pm}=1 \pm(1-y)^{2}$ 
In the quark-parton model, when we neglect the gluons, the structure functions become:

$$
\begin{aligned}
F_{2}^{+} & =x D+x \bar{U}, \\
F_{2}^{-} & =x U+x \bar{D}, \\
F_{L} & =0, \\
x F_{3}^{+} & =x D-x \bar{U}, \\
x F_{3}^{-} & =x U-x \bar{D} .
\end{aligned}
$$

The terms $x U, x D, x \bar{U}$ and $x \bar{D}$ denote the sum of parton distributions for up-type and down-type quarks and anti-quarks, respectively. ${ }^{1}$ The \pm superscript on $\sigma$ and $F$ corresponds to the sign of $W^{ \pm}$. Below the $b$-quark mass threshold, these sums are related to the quark distributions as follows:

$$
\begin{aligned}
& x U=x u+x c, \\
& x \bar{U}=x \bar{u}+x \bar{c}, \\
& x D=x d+x s, \\
& x \bar{D}=x \bar{d}+x \bar{s} .
\end{aligned}
$$

In the FFNS the charm-quark densities are zero. In the phasespace corners $y \rightarrow 0$ and $y \rightarrow 1$ and using the same quarkparton model approximation, we have the following asymptotic relations:

$$
\begin{array}{ll}
y \rightarrow 0: & \sigma_{\text {charm,CC }}^{ \pm}=F_{2}^{ \pm}=x D(x \bar{D})+x \bar{U}(x U), \\
y \rightarrow 1: & \sigma_{\text {charm, }, \mathrm{CC}}^{ \pm}=\frac{1}{2}\left(F_{2}^{ \pm} \mp x F_{3}^{ \pm}\right)=x \bar{U}(x U) .
\end{array}
$$

Thus the contribution from the strange-quark PDF is suppressed at high $y$.

\subsection{XFITTER implementation}

All calculations are interfaced in XFITTER and available with $\overline{\mathrm{MS}}$ heavy-quark masses. The reference value of the $\overline{\mathrm{MS}}$ charm mass is set to $m_{c}\left(m_{c}\right)=1.27 \mathrm{GeV}$ [43], and $\alpha_{s}$ is set to the value used for the corresponding PDF extraction: $\alpha_{s}\left(M_{Z}\right)=0.1191$ for ABMP16 and $\alpha_{s}\left(M_{Z}\right)=0.118$ for NNPDF3.1. The renormalization and factorization scales are chosen to be $\mu_{\mathrm{r}}^{2}=\mu_{\mathrm{f}}^{2}=Q^{2}$.

To estimate theoretical scale uncertainties, $\mu_{\mathrm{r}}$ and $\mu_{\mathrm{f}}$ are simultaneously varied up and down by a factor of two. In the case of the FONLL-B calculations, also the independent $\mu_{r}$ and $\mu_{f}$ variations are checked. Furthermore, the PDF uncertainties are propagated to the calculated theoretical predictions, while the uncertainties arising from varying the charm mass $m_{c}\left(m_{c}\right)=1.27 \pm 0.03 \mathrm{GeV}$ by one standard deviation

\footnotetext{
${ }^{1}$ In these expressions, we neglect the CKM mixing for brevity, but it is fully contained in the calculations.
}

are smaller than $1 \%$ and therefore neglected. In the FONLLB scheme, as a cross check, the calculation was performed with the pole charm mass $m_{c}^{\text {pole }}=1.51 \mathrm{GeV}$ which is consistent with the conditions of the NNPDF3.1 extraction [5]. The obtained theoretical predictions differ from the ones calculated with $m_{c}\left(m_{c}\right)=1.27 \mathrm{GeV}$ by less than $1 \%$. The total theoretical uncertainties are obtained by adding in quadrature scale and PDF uncertainties.

\section{Comparison of theoretical predictions}

We now provide some numerical comparisons of the heavyflavor schemes using their separate input conditions and associated PDF sets. Caution is necessary in these comparisons as the PDF sets are extracted with different input assumptions, data sets, and tolerance criteria; this is, in part, why we shall separately display the $\mu_{\mathrm{r}}, \mu_{\mathrm{f}}$ and PDF uncertainties in the following.

\subsection{Comparison of theoretical predictions in the FFNS A and FONLL-B schemes}

Figures 1, 2 and 3 show theoretical predictions for the FFNS A and FONLL-B schemes calculated as described in the previous sections with their total uncertainties. The FFNS A and FONLL-B results agree reasonably well within uncertainties in the bulk of the phase space. However, in

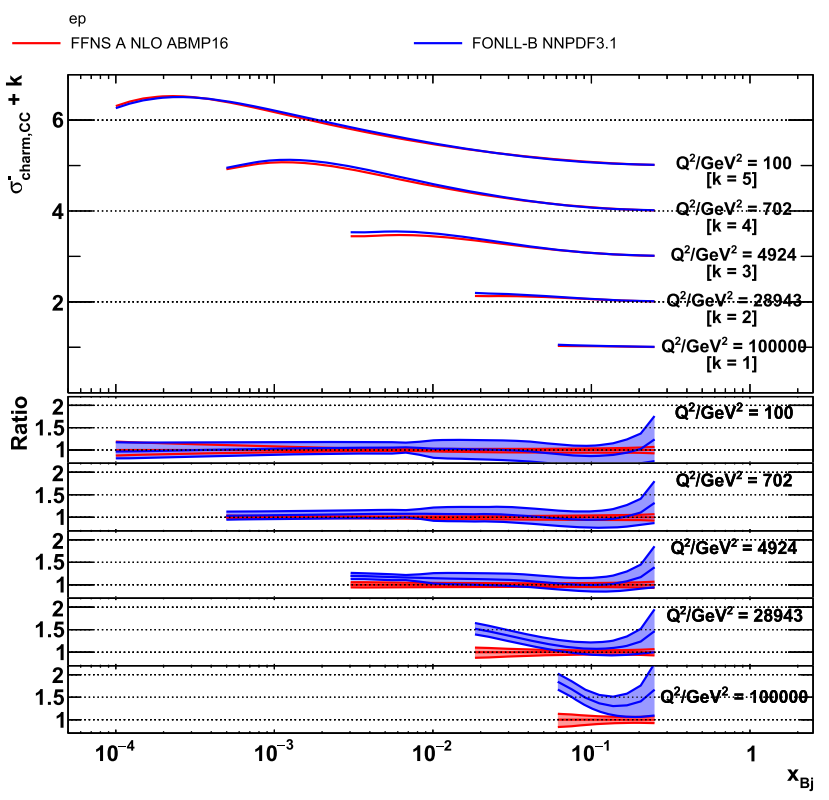

Fig. 1 The theoretical predictions with their total uncertainties for charm $\mathrm{CC}$ production at the $\mathrm{LHeC}$ as a function of $x_{\mathrm{Bj}}$ for different values of $Q^{2}$ calculated in the FFNS A and FONLL-B schemes. The bottom panels display the theoretical predictions normalized to the nominal values of the FFNS A predictions 


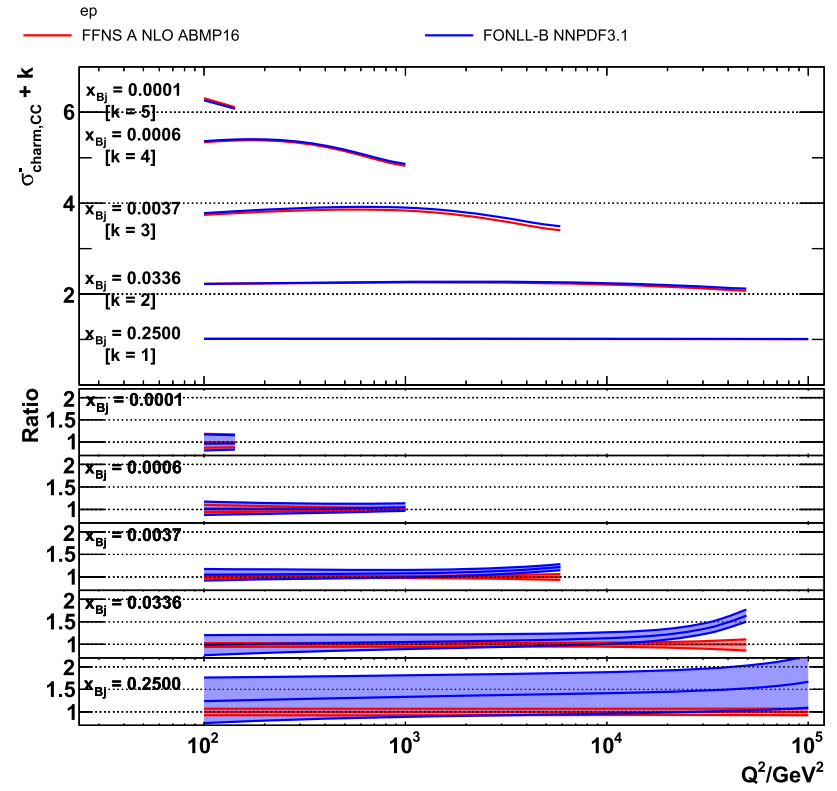

Fig. 2 The theoretical predictions with their total uncertainties for charm CC production at the $\mathrm{LHeC}$ as a function of $Q^{2}$ for different values of $x_{\mathrm{Bj}}$ calculated in the FFNS A and FONLL-B schemes. The bottom panels display the theoretical predictions normalized to the nominal values of the FFNS A predictions

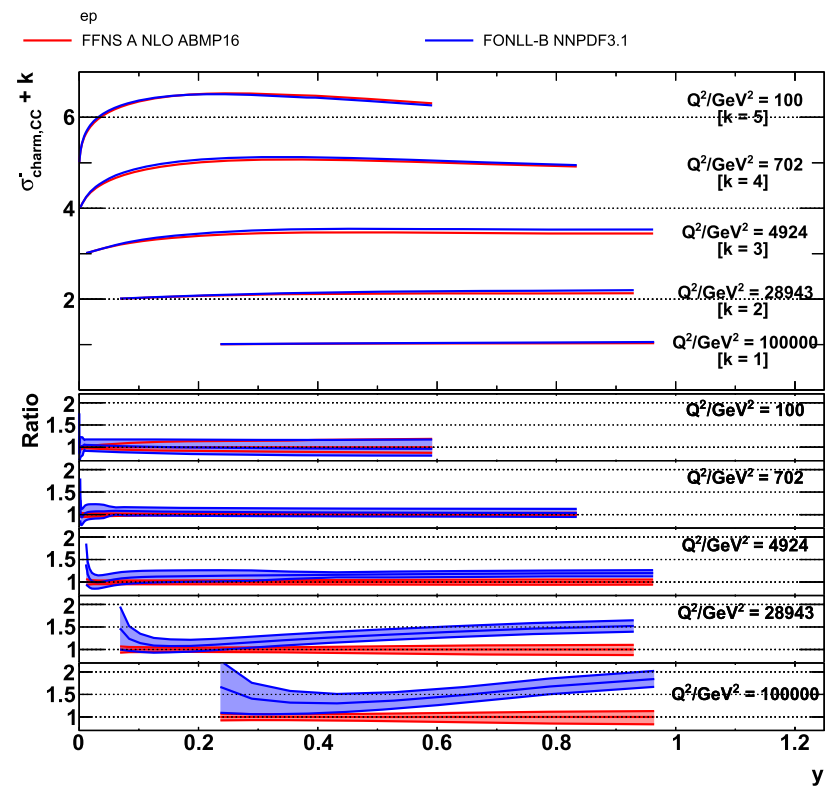

Fig. 3 The theoretical predictions with their total uncertainties for charm CC production at the $\mathrm{LHeC}$ as a function of $y$ for different values of $Q^{2}$ calculated in the FFNS A and FONLL-B schemes. The bottom panels display the theoretical predictions normalized to the nominal values of the FFNS A predictions

phase-space corners such as $Q^{2} \gtrsim 10000 \mathrm{GeV}^{2}$ or small $y$ the predictions in the two schemes differ by more than $50 \%$, exceeding the theoretical uncertainties.

To examine these differences further, in Fig. 4 we separately compute PDF and scale uncertainties (setting $\mu_{\mathrm{r}}=$ $\left.\mu_{\mathrm{f}}=\mu\right)$ of the charm CC cross section as a function of $Q^{2}$ for different values of $x_{\mathrm{Bj}}$ calculated in the FFNS A and FONLL-B scheme.

Comparing the two schemes, the larger variation of the FONLL-B scheme reflects the larger PDF uncertainty of the underlying PDF sets used: ABMP16 for FFNS A and NNPDF3.1 for FONLL-B. This difference is most evident in Fig. 4 which specifically separates out the PDF uncertainty, and reflects the independent inputs and assumptions used in the different PDF extractions.

Examining the results of Fig. 4, we also observe some other interesting features. For both of the calculations, the PDF uncertainties are relatively stable across the $Q^{2}$ range for fixed $x_{\mathrm{Bj}}$, but tend to increase at larger $x_{\mathrm{Bj}}$ values. As is well known, in pQCD calculations the effect of scale variations is indicative of the convergence of the series. We observe that the scale uncertainties for the FONLL-B scheme uniformly decrease with increasing $Q^{2}$. For the FFNS A scheme, the scale uncertainties decrease for small $x_{\mathrm{Bj}}$ values but increase with $Q^{2}$ at intermediate values of $x_{\mathrm{Bj}}$. Additional details are shown in Fig. 5 where we separately vary $\mu_{\mathrm{r}}$ and $\mu_{\mathrm{f}}$ for the FONLL-B scheme. Here we note that the uncertainty associated to $\mu_{\mathrm{r}}$ is very small and the total scale uncertainty is dominated by the variations of $\mu_{\mathrm{f}}$ which is tied to the PDFs, $f_{i}\left(x, \mu_{\mathrm{f}}\right)$. For the FFNS A in XFITTER, it is not possible to separately vary $\mu_{\mathrm{r}}$ and $\mu_{\mathrm{f}}$ in the current implementation, so the separate uncertainties can only be inferred by comparison to the FONLL-B case.

\subsection{Additional comparisons}

To further explore whether the differences between the two sets of theoretical predictions are due to the different treatment of heavy quarks or to the different PDF sets, theoretical calculations in FFNS A and FONLL-B are repeated with the HERAPDF2.0 PDF sets extracted from the HERA DIS data [2]. Predictions in the FFNS B scheme are also produced using the HERAPDF2.0 FF3B PDF set and the FFNS B matrix elements, which are equivalent to the FFNS A matrix elements at NLO for CC charm production. The results are displayed in Fig. 6. The differences between FFNS A and FONLL-B are similar to those displayed in Figs. 1, 2 and 3 and demonstrate that these differences arise from the different treatment of the heavy quarks in the two schemes. The FFNS B predictions lie between the FFNS A and FONLL-B predictions, indicating that a large part of the difference is due to the different treatment of heavy quarks in the running of $\alpha_{s}$ at high $x_{\mathrm{Bj}}$ or low $y$.

Furthermore, to investigate the impact of the NNLO corrections available at $Q \gg m_{c}$ for the FFNS calculation, approximate NNLO predictions are obtained using the ABMP16 NNLO PDF set [4]. The results for the cross section as a function of $Q^{2}$ for different values of $x_{\mathrm{Bj}}$ are shown in 

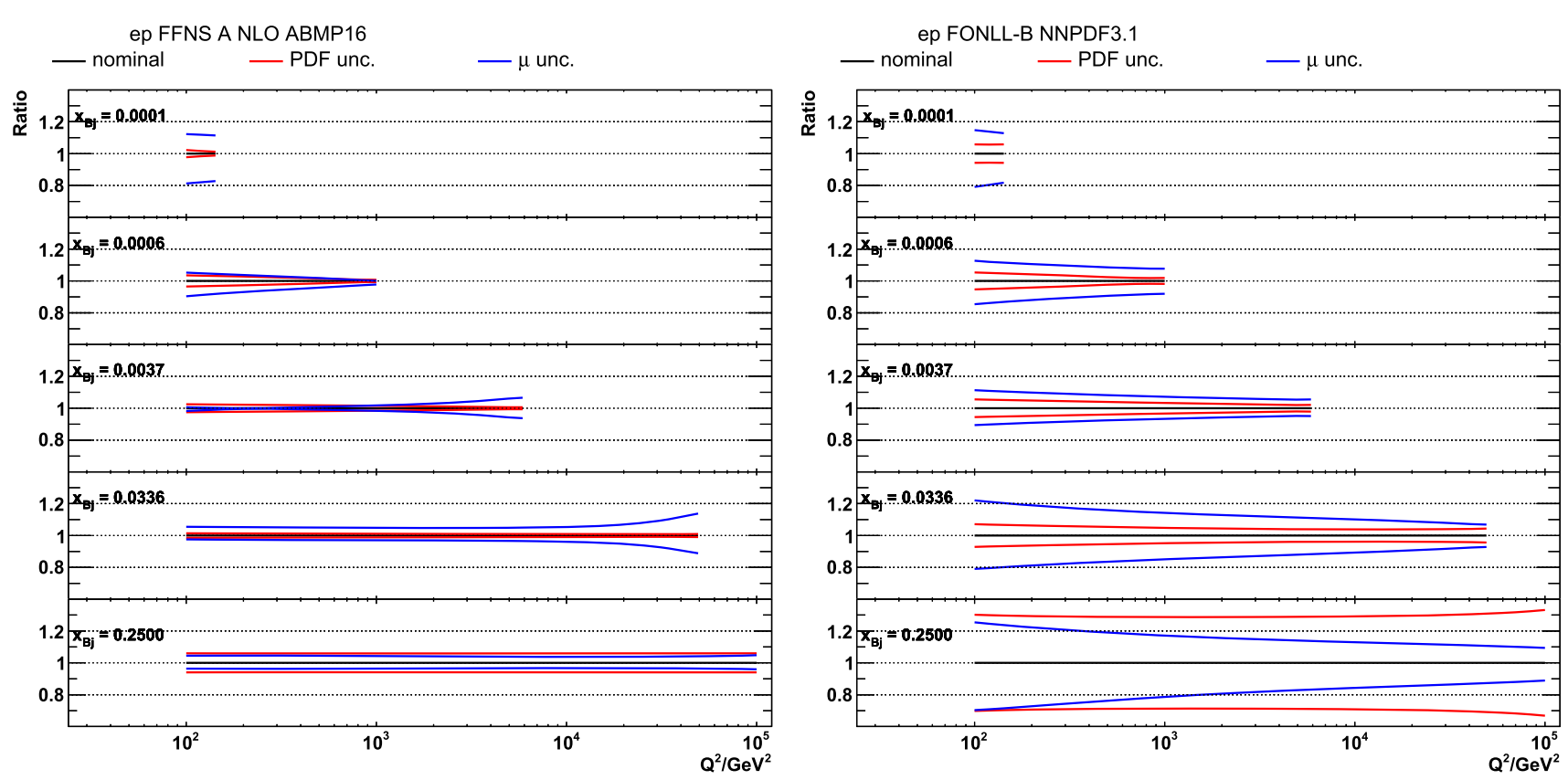

Fig. 4 Relative theoretical uncertainties of charm CC predictions for the LHeC as a function of $Q^{2}$ for different values of $x_{\mathrm{Bj}}$ calculated in the FFNS A and FONLL-B schemes. The PDF and scale uncertainties are shown separately
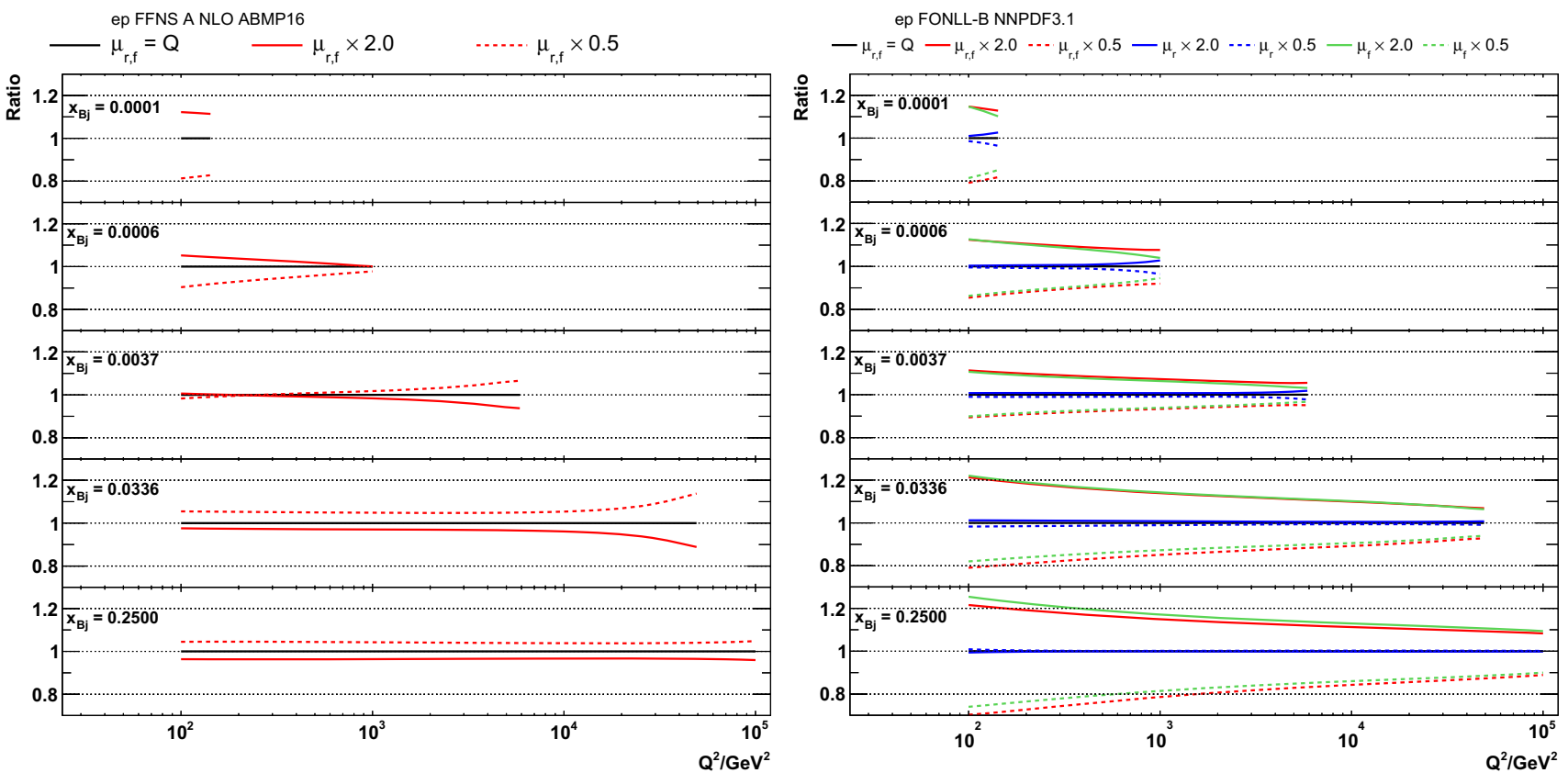

Fig. 5 The impact of separate scale variations on charm CC predictions for the LHeC as a function of $Q^{2}$ for different values of $x_{\mathrm{Bj}}$ calculated in the FFNS A and FONLL-B schemes

Fig. 7, where they are compared to the NLO FFNS A predictions from Fig. 2. The approximate NNLO corrections do not exceed $\sim 10 \%$ and thus cannot account for the differences between the FFNS A and FONLL-B theoretical predictions. Similar results are observed for the cross sections as functions of other kinematic variables.
To better understand the differences between the FFNS and VFNS calculations, Fig. 6 is particularly instructive. We see that at low $Q^{2}$ the FFNS (FFNS A and FFNS B) and VFNS (FONLL-B) results agree within uncertainties (as demonstrated in Fig. 2). When the scale $\mu$ is below the charmthreshold scale $\mu_{c}$ (typically taken to be equal to $m_{c}\left(m_{c}\right)$ ) the charm PDFs vanish and the FFNS and VFNS reduce to the 

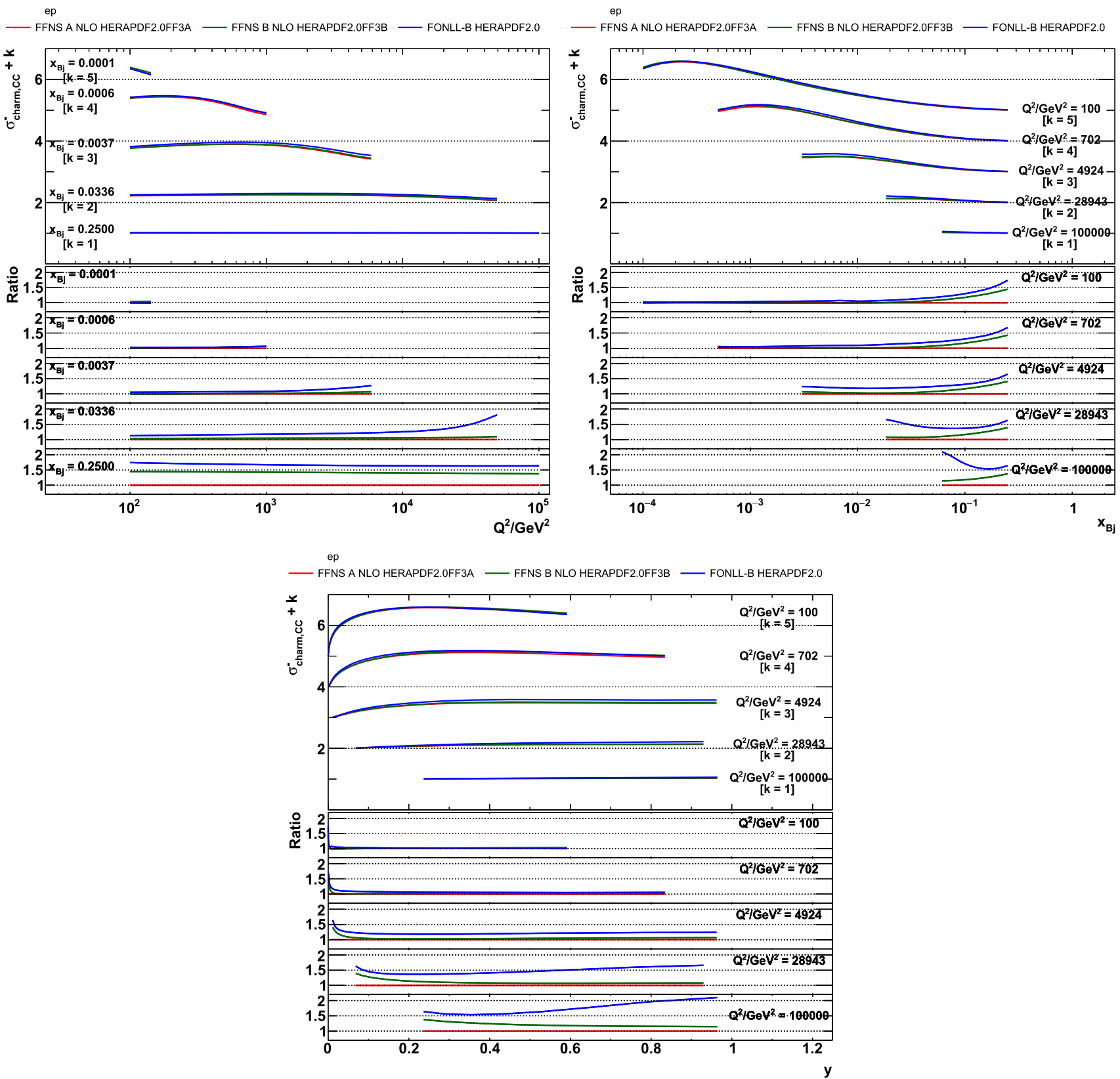

Fig. 6 The theoretical predictions for $\mathrm{CC}$ charm production at the $\mathrm{LHeC}$ as a function of $Q^{2}\left(x_{\mathrm{Bj}}, y\right)$ for different values of $x_{\mathrm{Bj}}\left(x_{\mathrm{Bj}}, Q^{2}\right)$ obtained using the HERAPDF2.0 PDF sets in the FFNS A, FFNS B and FONLL-B schemes. The bottom panels display the theoretical predictions normalized to the nominal values of the FFNS A predictions same result. ${ }^{2}$ For increasing scales, the VFNS resums the $\alpha_{s} \ln \left(\mu^{2} / \mu_{c}^{2}\right)$ contributions via the DGLAP evolution equations and the FFNS and VFNS will slowly diverge logarithmically. This behavior is observed in Fig. 6 and is consistent with the characteristics demonstrated in Ref. [52].

\footnotetext{
${ }^{2}$ Note that while the charm-threshold scale $\mu_{c}$ is commonly set to the charm quark mass $m_{c}\left(m_{c}\right)$, the choice of $\mu_{c}$ is arbitrary and amounts to a renormalization scheme choice [51].
}

More precisely, Ref. [52] used a matched set of $n_{f}=3$ and $n_{f}=5$ PDFs to study the impact of the scheme choice at large scales. They found that the resummed contributions in the VFNS yielded a larger cross section than the FFNS (the specific magnitude was $x$-dependent), and that for $Q^{2}$ scales more than a few times the quark mass, the differences due to scheme choice exceeded the differences due to (estimated) higher-order contributions. Thus, we have identified the source of the scheme differences at large $Q^{2}$. 


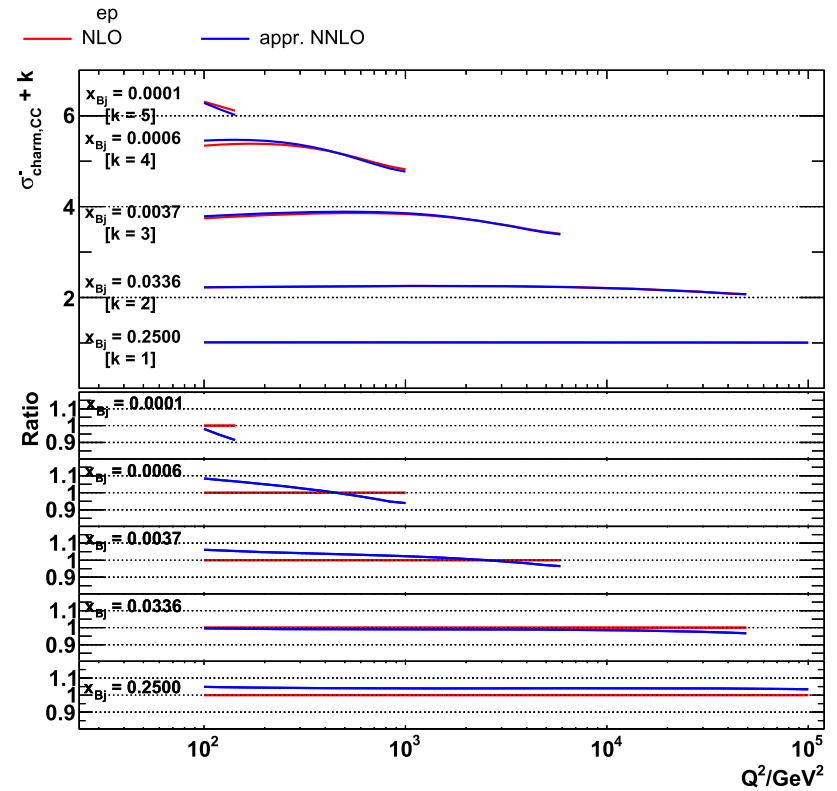

Fig. 7 The theoretical predictions for $\mathrm{CC}$ charm production at the LHeC as a function of $Q^{2}$ for different values of $x_{\mathrm{Bj}}$ calculated in the FFNS A scheme at NLO and approximate NNLO. The bottom panels display the theoretical predictions normalized to the nominal values of the FFNS A NLO predictions

The source of the scheme differences at large $x_{\mathrm{Bj}}$ is a bit more subtle. The VFNS includes a resummation of higherorder logarithms of the form $\alpha_{s} \ln \left(\mu^{2} / \mu_{c}^{2}\right)$. In Fig. 18 of the Appendix we display the separate contributions of the VFNS for a choice of $\left\{x_{\mathrm{Bj}}, Q^{2}\right\}$; the difference between the LO and SUB curves is indicative of the additional contribution of the resummed logarithms. This contribution depends on the particular $x_{\mathrm{Bj}}$ value as well as $Q^{2}$ (c.f., Fig. 11 of Ref. [52]). Thus, it is a balance between the LO and SUB contributions which determines the difference between the VFNS and FFNS; hence, the behavior is not just a simple factor. In Fig. 6b) we observe that for $Q^{2}$ values not too large $\left(Q^{2} \lesssim 4924 \mathrm{GeV}^{2}\right)$, the VFNS is above the FFNS A result for $x_{\mathrm{Bj}} \gtrsim 0.1$. Interestingly, we see the FFNS B scheme is quite similar to the VFNS in this kinematic region. For larger scales the patterns are not so clear, as the large $Q^{2}$ effects discussed previously now complicate the situation.

\subsection{Contributions from different partonic subprocesses}

The fundamental difference between the FFNS and the VFNS is the treatment of the heavy partons, the charm in particular. In the FFNS the charm is not included in the PDFs as an active parton, so charm quarks only arise from gluon splitting, $g \rightarrow c \bar{c}$. In contrast, the VFNS does include the charm as an active partonic flavor, and thus allows for charm-initiated subprocesses. To better appreciate these differences, we will study the individual partonic contributions to the cross section as functions of the kinematic variables $x_{\mathrm{Bj}}, Q^{2}$, and $y$.
Figures 8,9 and 10 show the contributions from separate partonic subprocesses to the $\mathrm{CC}$ charm production cross section in the FFNS A and FONLL-B schemes as a function of: $x_{\mathrm{Bj}}$ for different values of $Q^{2}, Q^{2}$ for different values of $x_{\mathrm{Bj}}$, and $y$ for different values of $Q^{2}$, respectively.

In these figures we observe that the gluon contribution to the FFNS is strikingly similar to the charm contribution to the VFNS. This is explained by the fact that in the FFNS the charm is present only in the final state and produced predominantly in the hard process $W^{+} g \rightarrow c \bar{s}$. In contrast, in the VFNS the charm is present also in the initial state and mainly produced by $g \rightarrow c \bar{c}$ collinear splitting through DGLAP evolution. The fundamental underlying process is (and has to be) the same in both the FFNS and VFNS, but the factorization boundary between PDFs and hard scattering cross section, $\hat{\sigma} \otimes f$, (determined by the scale $\mu$ and the scheme choice) is different. ${ }^{3}$

These figures highlight another interesting feature of the QCD theory; we observe that for the VFNS the gluon contribution (green curves) can become negative in particular kinematic regions. ${ }^{4}$ This is because in the VFNS we combine the gluon-boson fusion process (the NLO terms of Figs. 16 and 17) with the counter-term (the SUB terms), and this combination can be negative. This behavior underscores the fact that the renormalization scale $\mu$ is simply "shuffling" contributions among the separate sub-pieces, but the total physical cross section remains positive and stable, $c f$., Fig. 18 and Ref. [54]. This is a triumph of the QCD theory.

Next, turning our attention to the strange PDF contribution, it is notable that the FFNS and VFNS behave qualitatively very similar as functions of $Q^{2}, x_{\mathrm{Bj}}$, and $y$. In particular, we observe that the strange fraction increases for $x_{\mathrm{Bj}}$ and decreases for $Q^{2}$ and $y$. In particular, at high $y$ the strange PDF contribution drops to zero in favor of the gluon or charm quark PDFs (see Fig. 10 and Eq. (5)). Similar phenomena (although less pronounced) are observed at low $x_{\mathrm{Bj}}$ and/or high $Q^{2}$. In these phase-space regions, the dominant contributions to the cross section are proportional to the gluon PDF in the FFNS or to the charm-quark PDFs in the VFNS.

Finally, we note that in Fig. 9 for the FFNS A, the gluon contribution at high $x_{\mathrm{Bj}}\left(x_{\mathrm{Bj}} \sim 0.25\right)$ is minimal throughout the $Q^{2}$ range. To ensure this is not an artifact of either the FFNS A or the ABMP16 set, we regenerated these curves (not shown) in the FFNS B with the HERAPDF2.0 FF3B set and found the same behaviour; hence, this feature is truly a characteristic of the FFNS in the high- $x_{\mathrm{Bj}}$ region.

\footnotetext{
3 Note there is a "subtraction" term which closely matches the LO process, but this $\mathscr{O}\left(\alpha_{s}\right)$ process is contained in the NLO gluon-initiated contribution. For details, see Appendix A

4 Note, the FFNS can also have negative contributions at higher orders due to a similar "subtration" term for the strange PDF [53].
} 

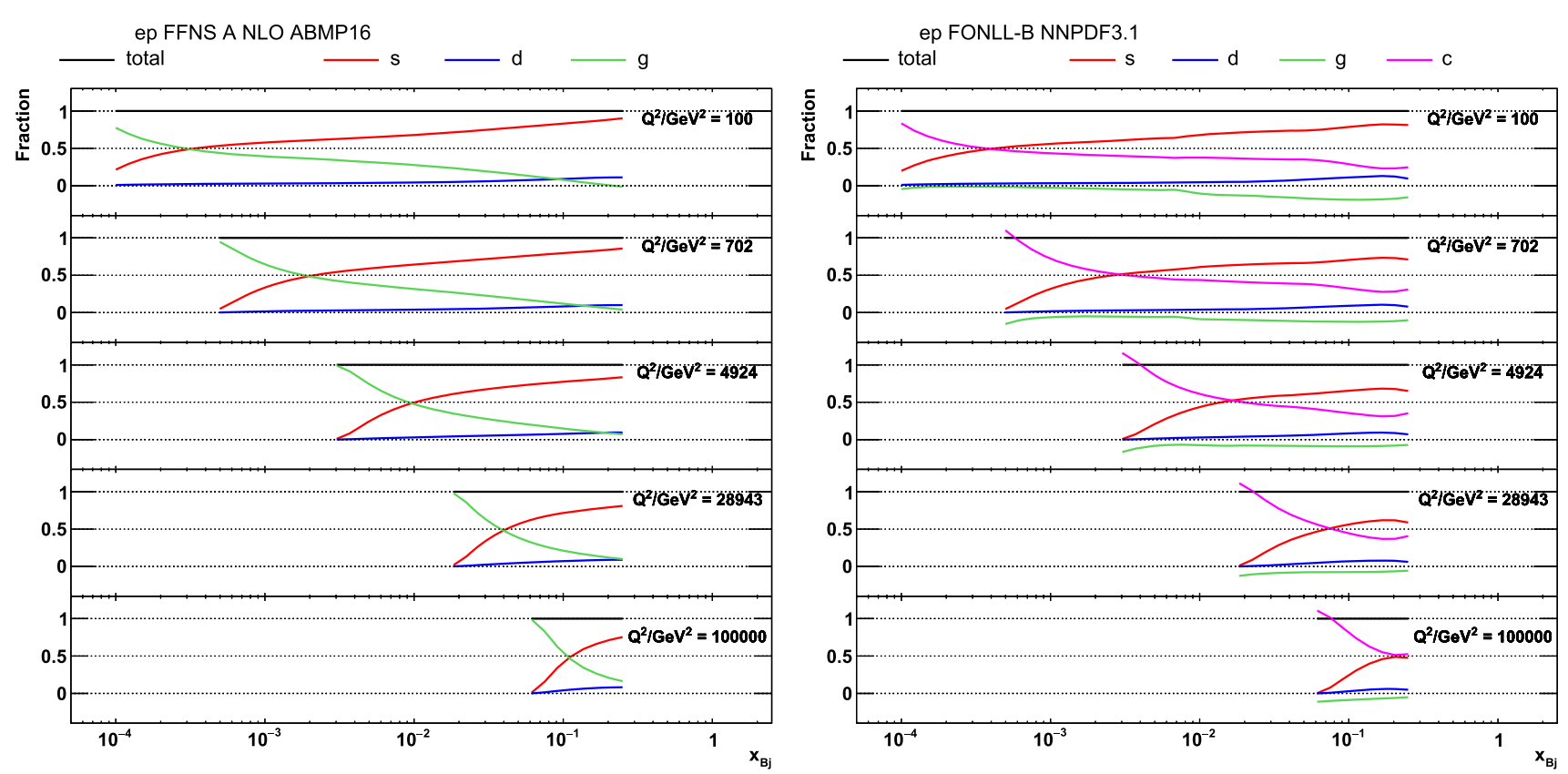

Fig. 8 The partonic subprocesses for charm CC production cross sections in the FFNS A (left) and FONLL-B (right) schemes as a function of $x_{\mathrm{Bj}}$ for different values of $Q^{2}$
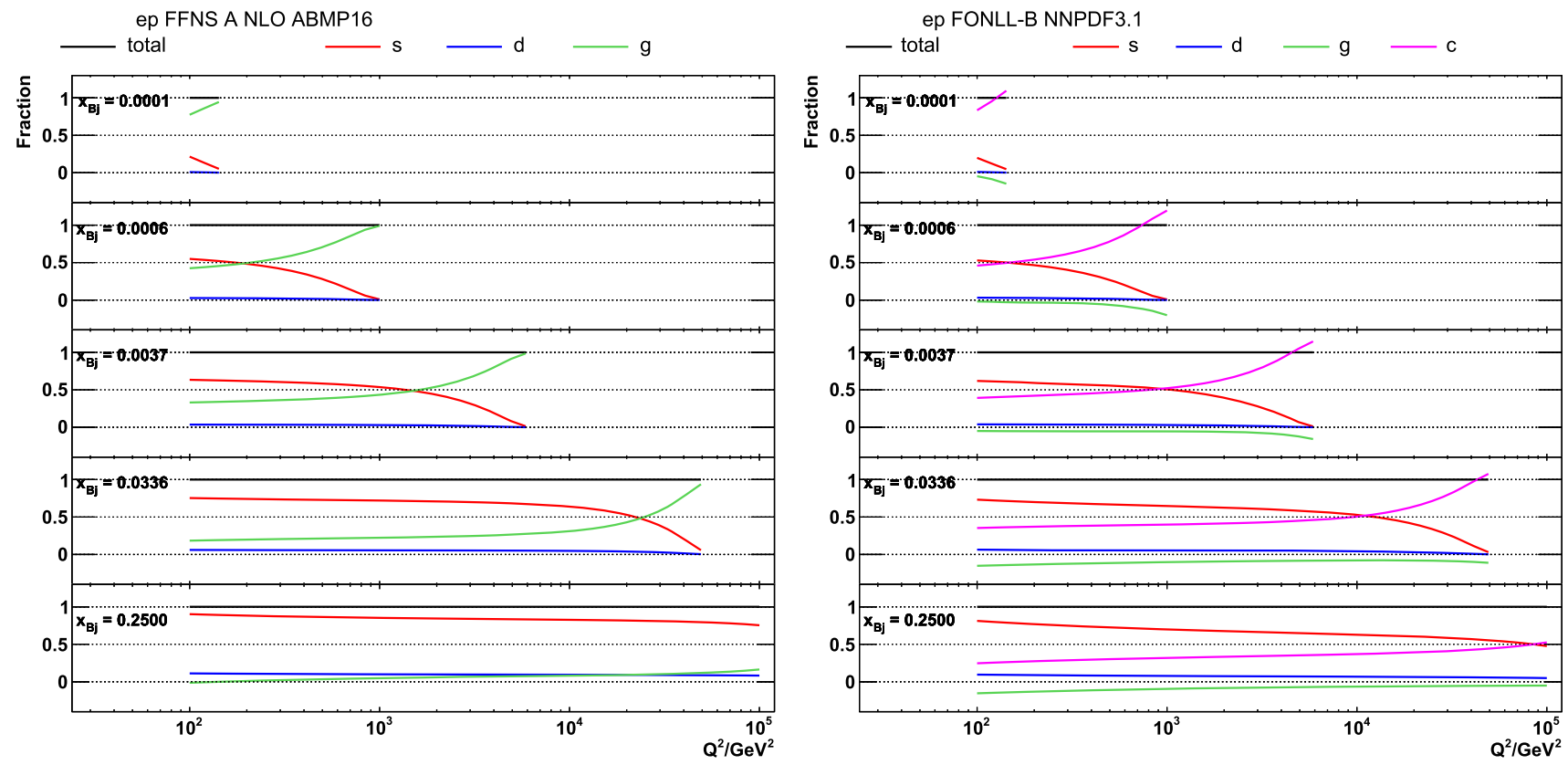

Fig. 9 The partonic subprocesses for charm CC production cross sections in the FFNS A (left) and FONLL-B (right) schemes as a function of $Q^{2}$ for different values of $x_{\mathrm{Bj}}$

\section{PDF constraints from charm CC pseudodata}

Now we turn to examine how the $\mathrm{LHeC}$ can reduce the PDF uncertainties and thus improve our predictive power.

The impact of charm CC cross section measurements at the $\mathrm{LHeC}$ on the PDFs is quantitatively estimated using the profiling technique [55]. This technique is based on minimizing the $\chi^{2}$ between data and theoretical predictions taking into account both experimental and theoretical uncertainties arising from PDF variations. Two NLO PDF sets were chosen for this study: ABMP16 [46] and NNPDF3.1 [5]. All PDF sets are provided with uncertainties in the format of eigenvectors. In the presence of strong constraints (the $\mathrm{LHeC}$ data is very precise), it is preferable to use the eigenvector representa- 

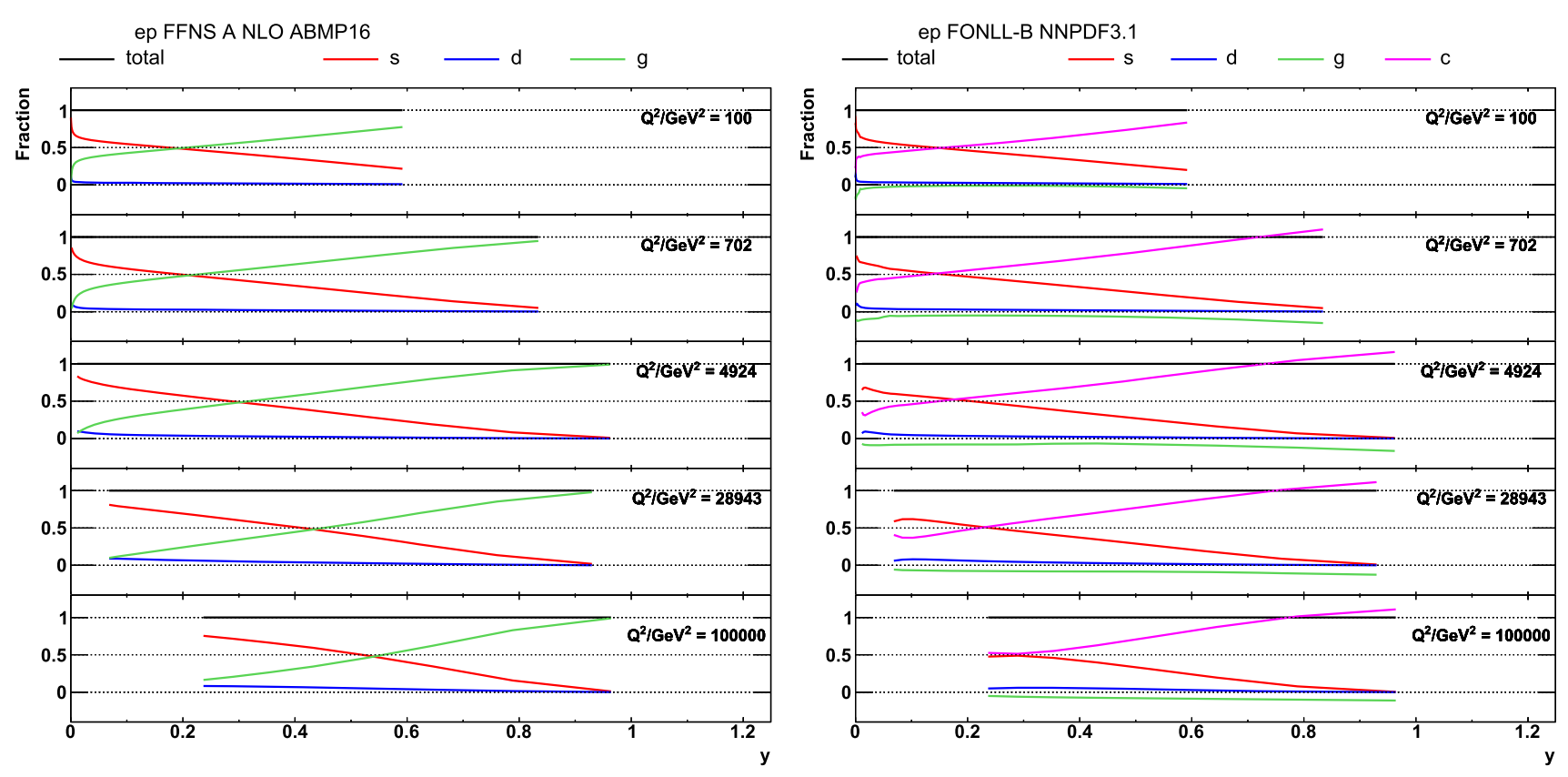

Fig. 10 The partonic subprocesses for charm CC production cross sections in the FFNS A (left) and FONLL-B (right) schemes as a function of $y$ for different values of $Q^{2}$

tion as only a few MC replicas would survive the Bayesian reweighting.

\subsection{The CC charm pseudodata}

For this study, pseudodata for charm CC production cross section differential in $Q^{2}$ and $x_{\mathrm{Bj}}$ and corresponding to an integrated luminosity of $100 \mathrm{fb}^{-1}[42,56]$ and polarization $P=-0.8$ are used. Theoretical predictions are calculated at NLO in pQCD both in the FFNS A with number of active flavors $n_{f}=3$ and in the FONLL-B scheme. The charmmass reference value in the $\overline{\mathrm{MS}}$ scheme is set to $m_{c}\left(m_{c}\right)=$ $1.27 \mathrm{GeV}$ and $\alpha_{s}$ is set to the value used for the corresponding PDF extraction. The renormalization and factorization scales are chosen to be $\mu_{\mathrm{r}}^{2}=\mu_{\mathrm{f}}^{2}=Q^{2}$.

The $\chi^{2}$ value is calculated as follows:

$$
\begin{aligned}
\chi^{2} & =\mathbf{R}^{T} \mathbf{C o v}^{-1} \mathbf{R}+\sum_{\beta} b_{\beta, \mathrm{th}}^{2}, \\
\mathbf{R} & =\mathbf{D}-\mathbf{T}-\sum_{\beta} \Gamma_{\beta, \mathrm{th}} b_{\beta, \mathrm{th}},
\end{aligned}
$$

where $\mathbf{D}$ and $\mathbf{T}$ are the column vectors of the measured (data) and predicted (theory) values, respectively. The correlated theoretical PDF uncertainties are included using the nuisance parameters $b_{\beta}$,th with their influence on the theory predictions described by $\Gamma_{\beta, \text { th }}$, where the index $\beta$ runs over all PDF eigenvectors. For each nuisance parameter a penalty term is added to the $\chi^{2}$, representing the prior knowledge of the parameter. No theoretical uncertainties except the PDF uncertainties are considered; the PDF Hessian uncertainties are treated symmetrically. The full covariance matrix Cov representing the statistical and systematic uncertainties of the data is used in the fit. The statistical and systematic uncertainties are treated as additive, i.e. they do not change in the fit. The systematic uncertainties are assumed uncorrelated between bins.

The values of the nuisance parameters at the minimum, $b_{\beta, \text { th }}^{\min }$, are interpreted as optimized, or profiled, PDFs, while uncertainties of $b_{\beta, \text { th }}^{\min }$ determined using the tolerance criterion of $\Delta \chi^{2}=1$ correspond to the new PDF uncertainties. The profiling approach assumes that the new data are compatible with the theoretical predictions using the existing PDFs, such that no modification of the PDF fitting procedure is needed. Under this assumption, the central values of the measured cross sections are set to the central values of the theoretical predictions.

\subsection{The profiled PDFs}

The profiling study is performed using two sets of LHeC charm CC pseudodata:

- the full set,

- a restricted set with data points for which the difference between the FFNS A and FONLL-B are smaller than the present $\mathrm{PDF}$ uncertainties. The latter is taken for simplicity as the sum of the ABMP16 and NNPDF3.1 uncertainties, but for the most data points it is dominated by the NNPDF3.1 uncertainties (see Fig. 4). 


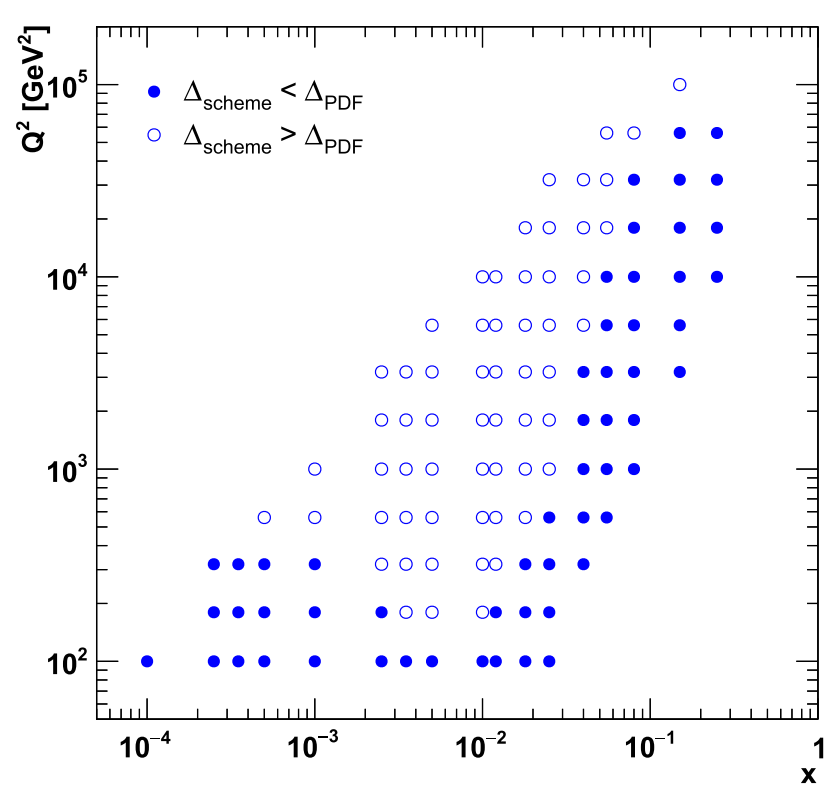

Fig. 11 The full $\left(\Delta_{\text {scheme }}<\Delta_{\mathrm{PDF}}, \Delta_{\text {scheme }}>\Delta_{\mathrm{PDF}}\right)$ and restricted $\left(\Delta_{\text {scheme }}<\Delta_{\mathrm{PDF}}\right)$ sets of data points which are used for PDF profiling

Given the sizable differences observed between the FFNS A and FONLL-B predictions, the study with the restricted data set (also referred to as 'with cuts') aims to check whether or not model independent constraints on the strange PDF can be extracted using the charm $\mathrm{CC}$ reaction at $\mathrm{LHeC}$. The two sets of data points are shown in Fig. 11 as functions of $Q^{2}$ and $x_{\mathrm{Bj}}$.

The comparison between ABMP16 and NNPDF3.1 is insightful as ABMP16 represents a more restricted parametrization. For the HERAPDF2.0 set, the strange PDF was not fit directly, but computed via the relation $f_{s}=$ $\bar{s} /(\bar{s}+\bar{d})=0.4 \pm 0.1$ and the uncertainty was approximated using the variation on $f_{s}$; hence, these uncertainties are not the same as the Hessian diagonalized eigenvectors, so we will not profile the HERAPDF2.0 PDF set.

The original and profiled ABMP16 and NNPDF3.1 PDF uncertainties are shown in Figs. 12, 13, 14 and 15. The uncertainties of the PDFs are presented at the scales $\mu_{\mathrm{f}}^{2}=100$ $\mathrm{GeV}^{2}$ and $\mu_{\mathrm{f}}^{2}=100000 \mathrm{GeV}^{2}$. A strong impact of the charm CC pseudodata on the PDFs is observed for both PDF sets. In particular, the uncertainties of the strange PDF are strongly reduced once the pseudodata are included in the fit. Also the gluon PDF uncertainties are decreased. Furthermore, in the case of the NNPDF3.1 set, the charm PDF uncertainties are reduced significantly. For all PDF sets, only small differences can be noticed between the PDF constraints obtained using the full or restricted set because the whole $x_{\mathrm{Bj}}$ range is covered in both cases (see Fig. 11) despite the fact that the number of data points in the restricted set is roughly half of the total number of data points.
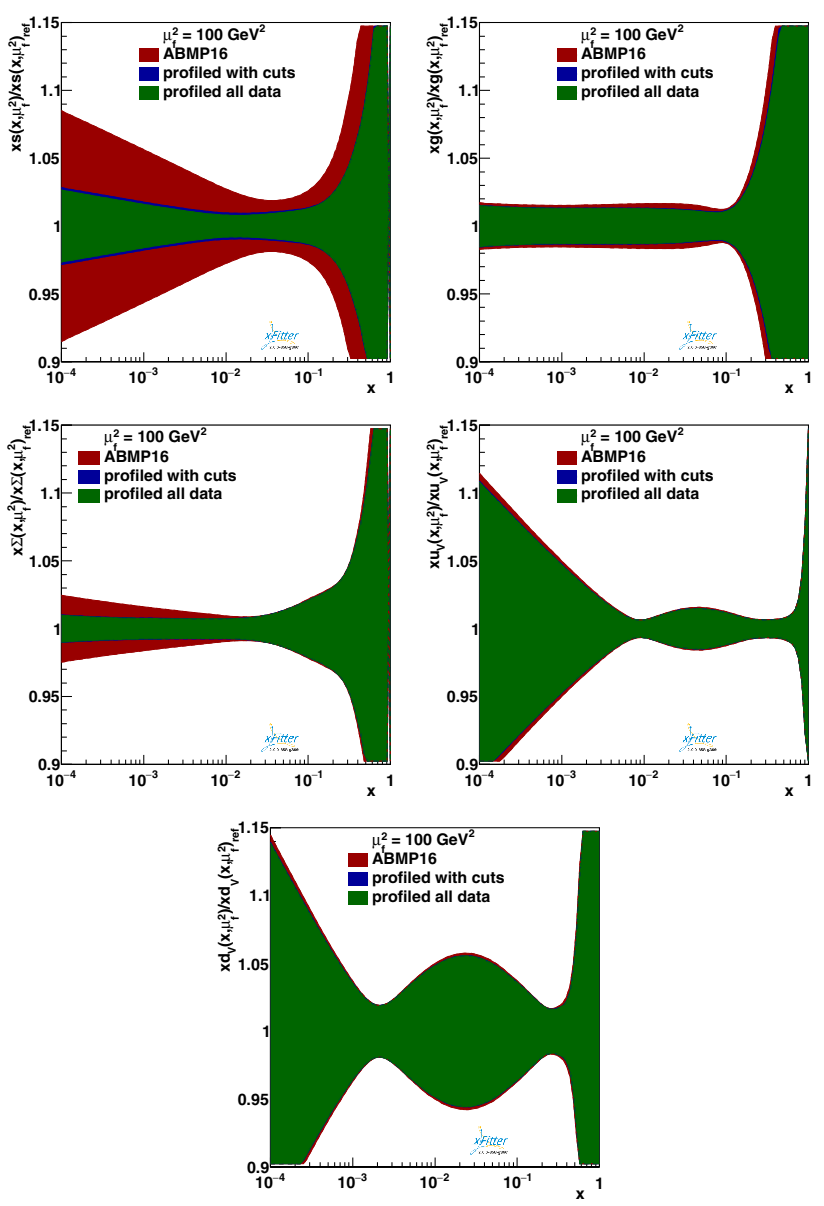

Fig. 12 The relative strange (top left), gluon (top right), sea quark (middle left), u valence quark (middle right) and d valence quark (bottom) PDF uncertainties at $\mu_{\mathrm{f}}^{2}=100 \mathrm{GeV}^{2}$ of the original and profiled ABMP16 PDF set

Additionally, in the case of the NNPDF3.1 set, it is possible to check the constraints on the strange quark and antiquark distributions separately, because no assumption $s=\bar{s}$ is used in NNPDF3.1. The $\mathrm{LHeC} e^{-} p$ pseudodata provide direct constraints only on $\bar{s}$. Nevertheless due to the apparently strong correlation between $s$ and $\bar{s}$ in the NNPDF3.1 fit, quite strong constraints are present on both the $s$ and $\bar{s}$ distributions once the direct constraints on $\bar{s}$ are provided by the LHeC pseudodata. However, only mild constraints are put on the ratio $s / \bar{s}$. This indicates that for precise determination of $s / \bar{s}$ both $e^{-} p$ and $e^{+} p$ data will be needed.

A recent study [25] has examined potential improvements in the PDFs for both the LHeC and HL-LHC facilities with both CC and NC. Our improvement in the strange PDF is comparable, while additional channels of Ref. [25] yields improved constraints on the gluon.

Comparing the results of profiled PDFs in the FFNS and the VFNS, we find both analyses are able to significantly improve the constraints on the strange quark PDF. This result 

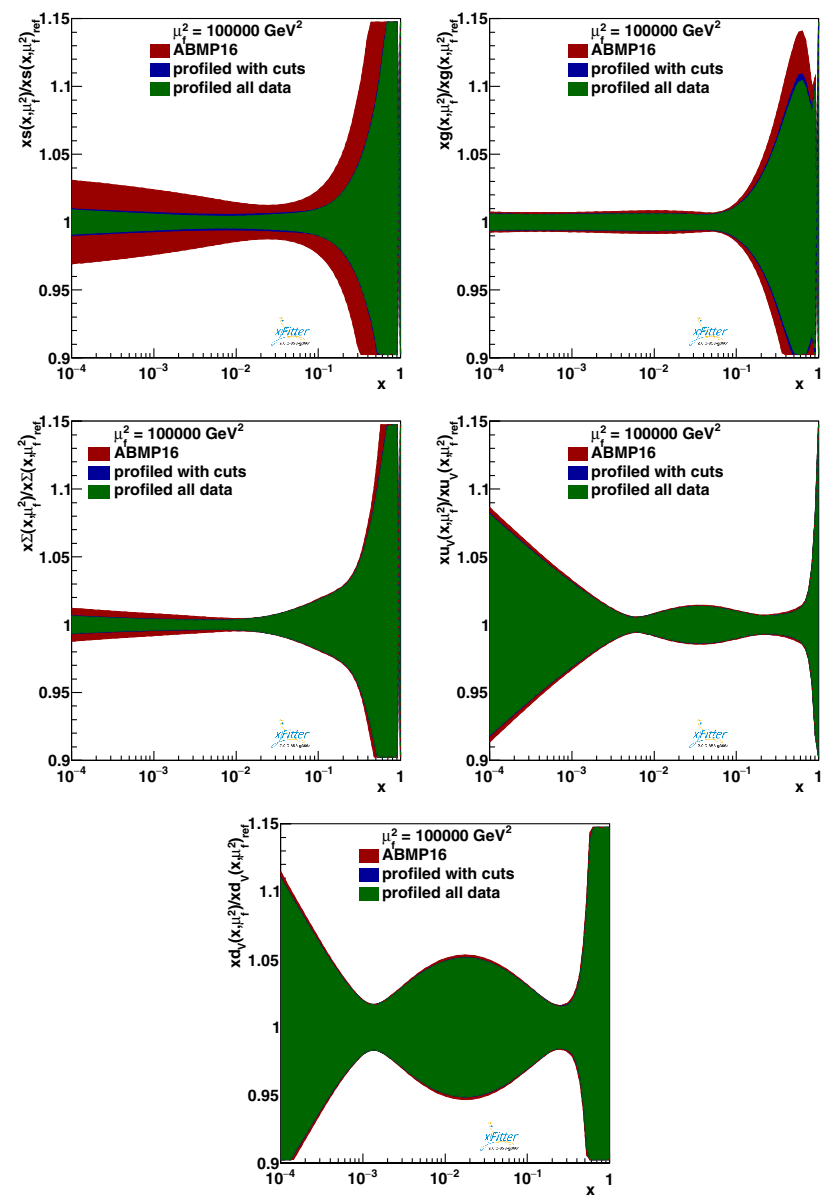

Fig. 13 The relative strange (top left), gluon (top right), sea quark (middle left), $\mathrm{u}$ valence quark (middle right) and $\mathrm{d}$ valence quark (bottom) PDF uncertainties at $\mu_{\mathrm{f}}^{2}=100000 \mathrm{GeV}^{2}$ of the original and profiled ABMP16 PDF set

gives us confidence that the general features we observe here are independent of the details of the heavy flavor scheme.

\section{Discussion and summary}

The recent performance of the LHC has exceeded expectations and produced an unprecedented number of precision measurements to be analyzed; thus, it is essential to improve the theoretical calculations to match. The uncertainty for many of these precision measurements stems primarily from the PDFs. Hence, our ability to measure fundamental parameters of the Standard Model (SM), such as the $W$ boson mass and $\sin ^{2} \theta_{W}$, ultimately comes down to how accurately we determine the underlying PDFs [57]. Additionally, our ability to characterize and constrain SM processes can indirectly impact beyond-standard-model (BSM) signatures.

We have focused on the strange-quark distribution which, at the LHC, can have a significant impact on the $W / Z$ cross
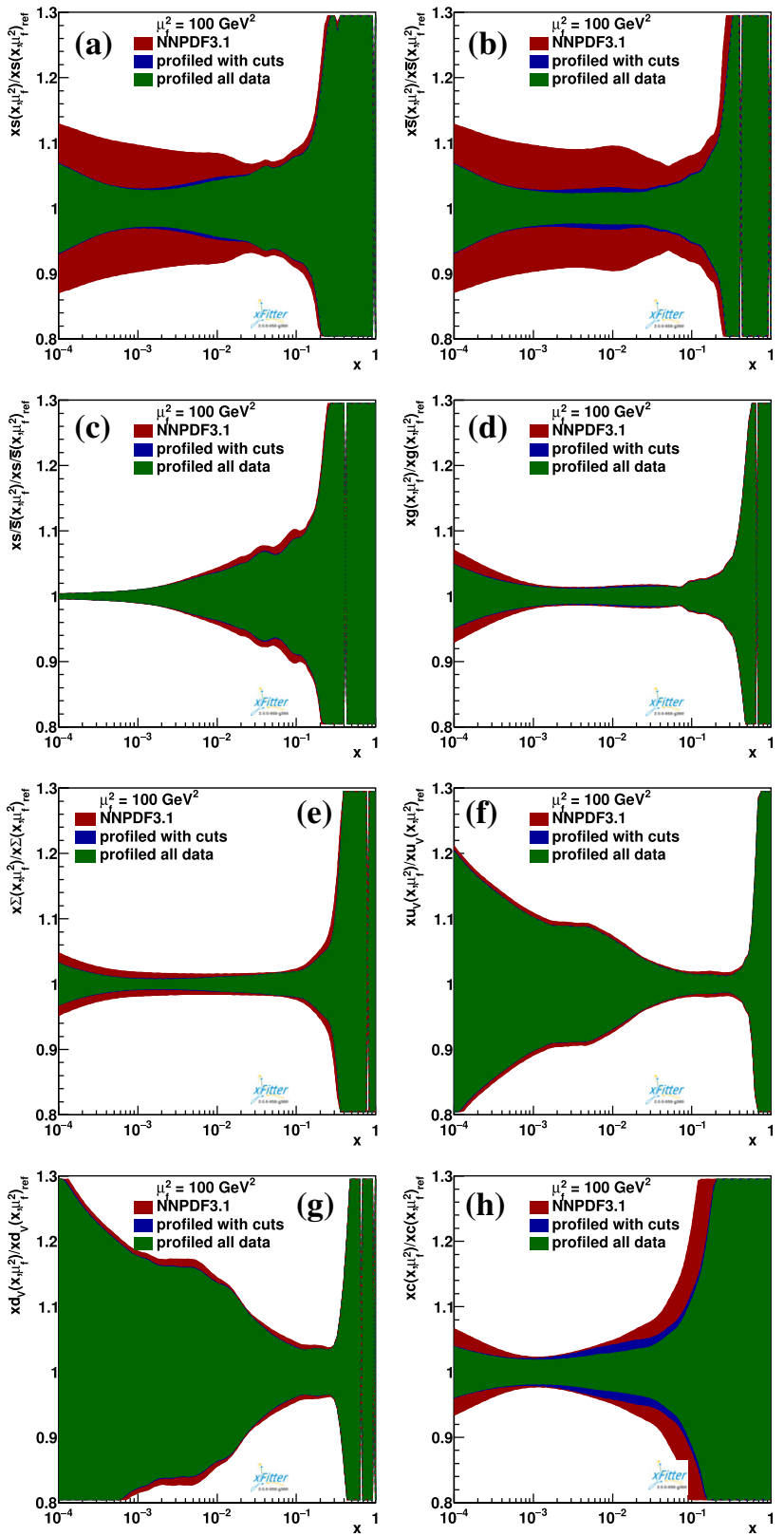

Fig. 14 The relative strange quark (a), strange anti-quark (b), and ratio $s / \bar{s}(\mathbf{c})$, gluon (d), sea quark (e), u valence quark $(\mathbf{f}), \mathrm{d}$ valence quark (g) and charm quark (h) PDF uncertainties at $\mu_{\mathrm{f}}^{2}=100 \mathrm{GeV}^{2}$ of the original and profiled NNPDF3.1 PDF set

section: one of the "standard candle" measurements. If we can reduce the uncertainty for these predictions, we can set stringent limits on any admixture of physics at higher scales. Unfortunately, at present the strange PDF has a comparably large uncertainty because measurements from the LHC and HERA, as well as older fixed-target experiments, do not seem to provide a definitive result for this flavor component.

This situation has prompted us to examine the CC DIS charm production at the $\mathrm{LHeC}$ to determine the impact of this data set on the PDF uncertainty. We considered the LHeC 

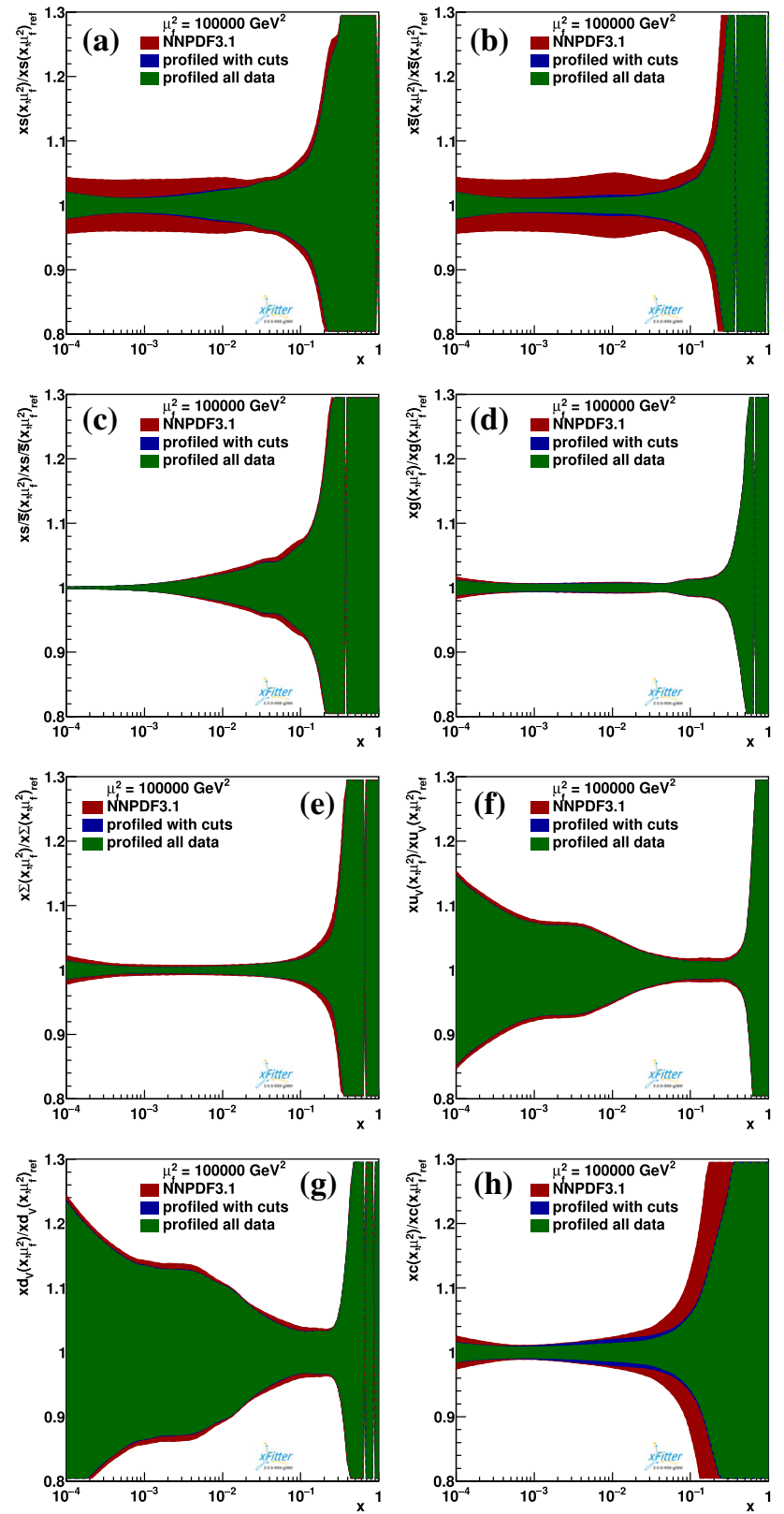

Fig. 15 The relative strange quark (a), strange anti-quark (b), and ratio $s / \bar{s}(\mathbf{c})$, gluon (d), sea quark (e), u valence quark (f), d valence quark (g) and charm quark (h) PDF uncertainties at $\mu_{\mathrm{f}}^{2}=100000 \mathrm{GeV}^{2}$ of the original and profiled NNPDF3.1 PDF set

as this high-energy ep/A facility could potentially run in parallel with the LHC and provide insights into these issues at low $x$ and high $Q^{2}$ in advance of a FCC program.

This case study of the CC DIS charm production at the $\mathrm{LHeC}$ provides a practical illustration of the many features of XFITTER. As the XFITTER framework is designed to be a versatile open-source software framework for the determination of PDFs and the analysis of QCD physics, we can readily adapt this tool to address the impact and influence of new data sets. Furthermore, as both FFNS and VFNS calculations are implemented, we can use XFITTER as a theoretical "laboratory" to study the resummation of large logarithms and multi-scale issues. We have outlined some of these issues in the Appendix. In particular, the CC DIS charm production involves a flavor-changing $W^{ \pm}$boson, multiple quark masses enter the calculation, and this introduces some subtle theoretical issues to properly address the disparate mass and energy scales.

Using the XFITTER framework, we find that the LHeC can provide strong constraints on the strange-quark PDF, especially in the previously unexplored small- $x_{\mathrm{Bj}}$ region. ${ }^{5}$ A large reduction of uncertainties is observed also when restricting the input data to the kinematic range where the differences between the FFNS A and FONLL-B schemes are not larger than the present PDF uncertainties, indicating that the obtained PDF constraints are stable and independent of the particular heavy-flavor scheme. As noted above, a reduction of the strange-PDF uncertainties influences the $W / Z$ production, and thus the Higgs production; hence, the LHeC CC DIS charm production data represent a valuable addition for the future global PDF fits.

However, since charm CC production in $e^{-} p$ collisions mostly probe $\bar{s}$, only mild constraints are put on the ratio $s / \bar{s}$ using the NNPDF3.1 PDF set as reference; therefore for a precise determination of this ratio, both $e^{-} p$ and $e^{+} p$ data will be needed.

In conclusion, we find that $\mathrm{CC}$ DIS charm production at the $\mathrm{LHeC}$ can provide strong constraints on the strange PDF which are complementary to the current data sets. As the PDF uncertainty is the dominant factor for many precision analyses, a reduction of these uncertainties will allow for more accurate predictions which can be used to constrain both SM and BSM physics processes.

Acknowledgements We would like to thank Max Klein for providing the pseudodata, and John C. Collins, Aleksander Kusina, Ted C. Rogers, Ingo Schienbein, George Sterman, for useful discussion. The work of O.Z. has been supported by Bundesministerium für Bildung und Forschung (contract $05 \mathrm{H} 18 \mathrm{GUCC} 1$ ). This work F. O. was supported by the U.S. Department of Energy under Grant No. DE-SC0010129.

Data Availability Statement This manuscript has associated data in a data repository. [Authors' comment: The xFitter program and the associated data files are available for download from http://www.xfitter. $\operatorname{org} /$.

Open Access This article is distributed under the terms of the Creative Commons Attribution 4.0 International License (http://creativecomm ons.org/licenses/by/4.0/), which permits unrestricted use, distribution, and reproduction in any medium, provided you give appropriate credit to the original author(s) and the source, provide a link to the Creative Commons license, and indicate if changes were made. Funded by SCOAP $^{3}$.

\footnotetext{
${ }^{5}$ In this study we have focused exclusively on the $\mathrm{LHeC}$ result for $\mathrm{CC}$ DIS charm production; however, the $\mathrm{LHeC}$ has a broad multi-faceted program which is described in Refs. [58,59].
} 


\section{Appendix A: $F_{2}^{c}$ Beyond leading-order}

The multi-scale problem: The CC DIS charm production process involves some interesting issues that we will explore here in detail. In particular, there are multiple mass and energy scales which span a wide kinematic range, and it becomes an intricate puzzle to treat them all properly.

For this current illustration, we will focus on the contribution to the DIS $F_{2}^{c}$ structure function from the process involving the strange and charm quark; other quark combinations can be addressed in a similar manner. The fully inclusive $F_{2}$ can be studied using the energy and angle of the outgoing lepton; in contrast, $F_{2}^{c}$ also requires information about the final hadronic state, and this introduces some subtleties. In particular, we will show that as we go to higher orders the $F_{2}^{c}$ structure function must be defined carefully so that: i) theoretically it is free of divergences and independent of the renormalization scales when calculated to all orders, and ii) experimentally it matches what is measured by the detector.

The mass scales: What makes this process complex is that we encounter a number of different mass scales. Furthermore, there is no fixed hierarchy for the mass scales, and we will need to compute both in the low- $Q$ region, where $Q \lesssim m_{c}$, as well as in the high- $Q$ region, $Q \gg m_{c}$.

The $Q$ scale is related to the invariant mass of the virtualboson probe ( $W^{+}$in this case), and can be expressed in terms of the energy and angle of the lepton; this is a physically measurable kinematic variable.

In contrast, the scale $\mu$ is an unphysical scale which implements the separation between the PDF and the hard-scattering cross section, and the scale at which $\alpha_{s}$ is evaluated; thus, the physics should be insensitive to a variation of $\mu$. As our calculations typically involve the dimensionless combination $\ln (\mu / Q)$, we generally choose $\mu \sim Q$ to avoid large logarithms.

The strange quark is a "light" active parton with an associated PDF $s(x)$ and mass $m_{s}<\Lambda_{\mathrm{QCD}}$. The strange-quark mass is comparable to or less than other hadronic scales which are neglected; as such, it serves only as a regulator and plays no physical role. Effectively, we can take $m_{s} \rightarrow 0$ if we choose. We treat the up and down quarks masses $m_{u, d}$ in a similar manner.

The charm quark is a "heavy" object; its associated mass $m_{c}>\Lambda_{\mathrm{QCD}}$ does play a physical role and cannot generally be neglected. There may or may not be a PDF associated with the charm. In a $n_{f}=3$ FFNS scheme, we will assume the charm PDF to be zero. ${ }^{6}$ In a VFNS there is a charm PDF only when the $\mu$ scale is above the scale where the charm PDF is activated; we call this the matching scale, $\mu_{c}$. It is

\footnotetext{
${ }^{6}$ It is possible to extend this to incorporate an intrinsic-charm PDF.
}

common $^{7}$ to set $\mu_{c}=m_{c}$, but this is not required. ${ }^{8}$ In this study, however, we will adopt this common choice.

Because there are two different quark masses involved $\left(m_{s}\right.$ and $m_{c}$ ) in the CC DIS process, we can examine the mass singularities of the $t$-channel and $u$-channel separately. This separation is particularly useful to understand how the individual mass singularities are addressed, and how the FFNS and the VFNS organize the contributions to the total structure function.

The $n_{f}=3$ FFNS: To be specific, we will consider CC DIS production of a charm quark. We first compute this in the $n_{f}=3$ FFNS where $\{u, d, s\}$ are light "active" partons in the proton, and the charm $c$ is considered an external "heavy" particle. This can be implemented in the ACOT scheme [54] for example by using a CWZ renormalization [62] where the light "active" partons are renormalized with normal $\overline{\mathrm{MS}}$, and the "heavy" quarks use a zero-momentum subtraction. In this scheme, the leading-order (LO) process is $s W^{+} \rightarrow c$ as illustrated in Fig. 16. At next-to-leading-order (NLO), we then include $g W^{+} \rightarrow c \bar{s}$ which has both $t$-channel (Fig. 16) and $u$-channel (Fig. 17) contributions. ${ }^{9}$

$t$-channel: The $t$-channel process has an intermediate $s$ quark exchanged, and if we use the strange quark mass $m_{s}$ to regulate the singularities, this will yield a contribution proportional to $\ln \left(Q / m_{s}\right)$. This mass singularity arises from the region of phase space where the exchanged $s$-quark becomes collinear and close to the mass shell; that is, when the phase space of the $g W^{+} \rightarrow c \bar{s}$ process begins to overlap with that of the $s W^{+} \rightarrow c$ process. This "double counting" is resolved by a subtraction (SUB) counter-term given by:

$(S U B) \sim f_{g} \otimes \widetilde{f}_{g \rightarrow s} \otimes \sigma_{s W^{+} \rightarrow c}$.

Here, $\tilde{f}_{g \rightarrow s}$ is the perturbative splitting of the gluon into an $s \bar{s}$ pair; the leading term is proportional to: ${ }^{10}$

$$
\tilde{f}_{g \rightarrow s}(x, \mu) \sim \frac{\alpha_{S}(\mu)}{2 \pi} P_{g \rightarrow s}^{(1)}(x) \ln \left(\frac{\mu^{2}}{m_{s}^{2}}\right)+\mathscr{O}\left(\alpha_{s}^{2}\right)
$$

\footnotetext{
7 The choice of matching scale $\mu_{c}=m_{c}$ is common because at NLO the $\overline{\mathrm{MS}}$ matching conditions on the PDFs are proportional to the DGLAP kernel times $\ln \left(\mu / m_{c}\right)$. As an explicit calculation shows, the constant term vanishes. Therefore, by choosing $\mu_{c}=m_{c}$ we have the simple boundary condition $f_{c}\left(x, \mu=m_{c}\right)=0$. At NNLO, the constant term is non-zero and this yields $f_{c}\left(x, \mu=m_{c}\right) \neq 0$. See Ref. [60] and references therein.

${ }^{8}$ By displacing the matching scale to larger values $\mu_{c}>m_{c}$, one can have the advantage of avoiding delicate cancellations in the region $\mu \sim m_{c}$; this flexibility was explored in Refs. [51,61].

${ }^{9}$ Note, there are also corresponding quark-initiated processes; we will focus on the gluon-initiated processes as this is sufficient to illustrate our points. Both the gluon- and quark-initiated contributions are included in our calculations.

10 The scale of the SUB term is $\mu$ as the relevant scale here is the renormalization scale of the PDF: $f(x, \mu) \otimes \hat{\sigma}(x, Q, \mu)$.
} 
Fig. 16 The $t$-channel processes up to $\mathscr{O}\left(\alpha_{S}^{1}\right)$. Note we sum the combination (NLO-SUB) to obtain the complete $\mathscr{O}\left(\alpha_{S}^{1}\right)$ correction; we find it useful to study these terms separately. The higher-order quark-initiated contributions are not show, but are included in the calculation

Fig. 17 The $u$-channel processes up to $\mathscr{O}\left(\alpha_{S}^{1}\right)$. Note the NLO $t$-channel and $u$-channel terms are combined coherently at the amplitude level. The higher-order quark-initiated contributions are not show, but are included in the calculation
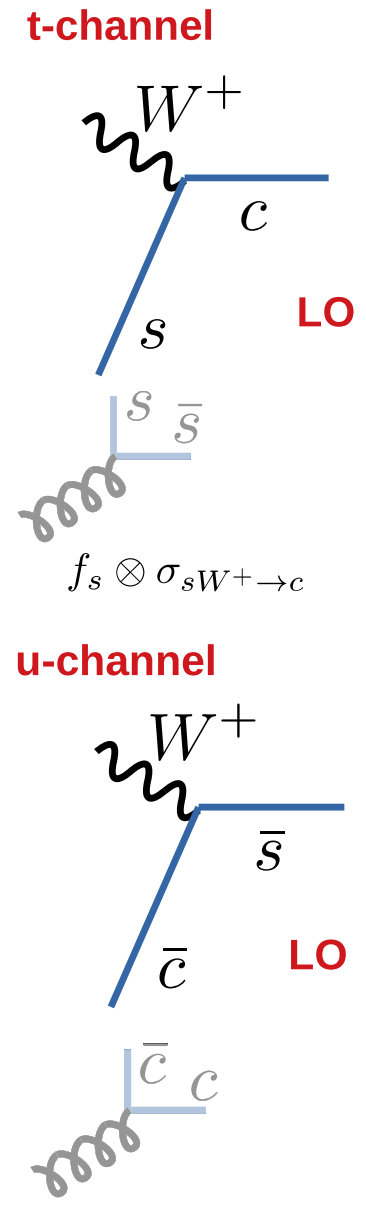

$f_{\bar{c}} \otimes \sigma_{\bar{c} W^{+} \rightarrow \bar{s}}$ gluon initiated

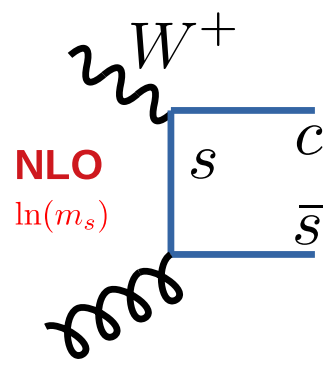

$f_{g} \otimes \sigma_{g W^{+} \rightarrow c \bar{s}}$

gluon initiated

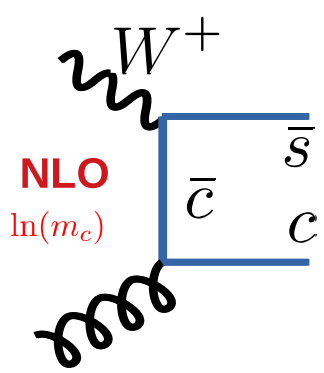

$f_{g} \otimes \sigma_{g W^{+}} \rightarrow \bar{s} c$ gluon initiated

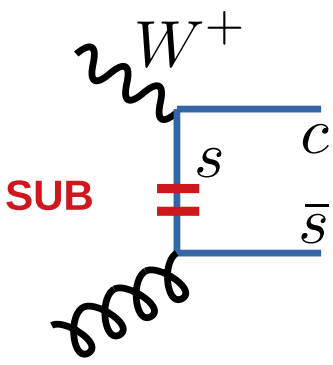

$\widetilde{f} \sim \ln \left(m_{s}\right)$

$f_{g} \otimes \widetilde{f}_{g \rightarrow s} \otimes \sigma_{s W^{+} \rightarrow c}$

gluon initiated

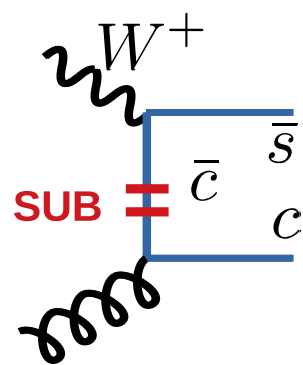

$\tilde{f} \sim \ln \left(m_{c}\right)$

$f_{g} \otimes \widetilde{f}_{g \rightarrow \bar{c}} \otimes \sigma_{\bar{c} W^{+} \rightarrow \bar{s}}$

where $P_{g \rightarrow s}^{(1)}(x)$ is the $\mathscr{O}\left(\alpha_{s}\right)$ DGLAP splitting kernel for $g \rightarrow s$.

The complete contribution to the structure function is given by:

$F_{2}^{c} \sim T O T=L O+(N L O-S U B)$

The complete $\mathscr{O}\left(\alpha_{s}\right)$ contribution is the combination $(N L O-S U B)$; our separation into $N L O$ and $S U B$ is simply to illustrate the interplay of these components. Both the NLO and SUB terms have $\ln \left(m_{s}\right)$ divergences, but these precisely cancel and yield a well-defined result even if we take the $m_{s} \rightarrow 0$ limit. ${ }^{11}$

$u$-channel: We next examine the $u$-channel NLO contribution to the $g W^{+} \rightarrow c \bar{s}$ process. This has an intermediate $c$-quark exchanged and is proportional to $\ln \left(Q / m_{c}\right)$. In the FFNS where the charm is a "heavy" non-parton, there is no counter-term for this graph, and the resulting observables will retain the $\ln \left(Q / m_{c}\right)$ dependence. In principle, this means that when we go to large $Q$ scales, these terms will begin to

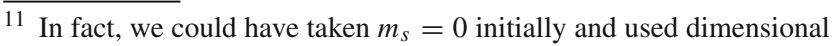
regularization to compute the contributions.
}

degrade the convergence of the perturbative series. In practice, while this degradation only grows logarithmically, at large scales (such as at the LHC energies) we do find it convenient to treat the charm on an equal footing as the $u, d, s$ partons.

The VFNS: We now turn to the VFNS scheme where we include the charm quark as an "active" parton and compute its associated PDF.

In this case, there is a $u$-channel counter-term (SUB) given by $f_{g} \otimes \widetilde{f}_{g \rightarrow \bar{c}} \otimes \sigma_{\bar{c} W^{+} \rightarrow \bar{s}}$ which is proportional to $\ln \left(\mu / m_{c}\right)$. The NLO $u$-channel contribution will have a $\ln \left(Q / m_{c}\right)$ factor, so the combination $(N L O-S U B)$ is also free of mass singularities. ${ }^{12}$

What is less obvious is that we must also include the LO process $\bar{c} W^{+} \rightarrow \bar{s}$. There are two ways we can understand why this is necessary.

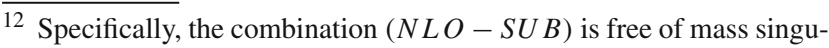
larities and finite in the limit $m_{c} \rightarrow 0$. Note that the VFNS fully retains the charm quark mass $m_{c}$ and (in contrast to some claims in the literature) the factorization holds up to $\mathscr{O}\left(\Lambda^{2} / Q^{2}\right)$ corrections; all terms of order $\left(m_{c}^{2} / Q^{2}\right)$ are fully included [63].
} 


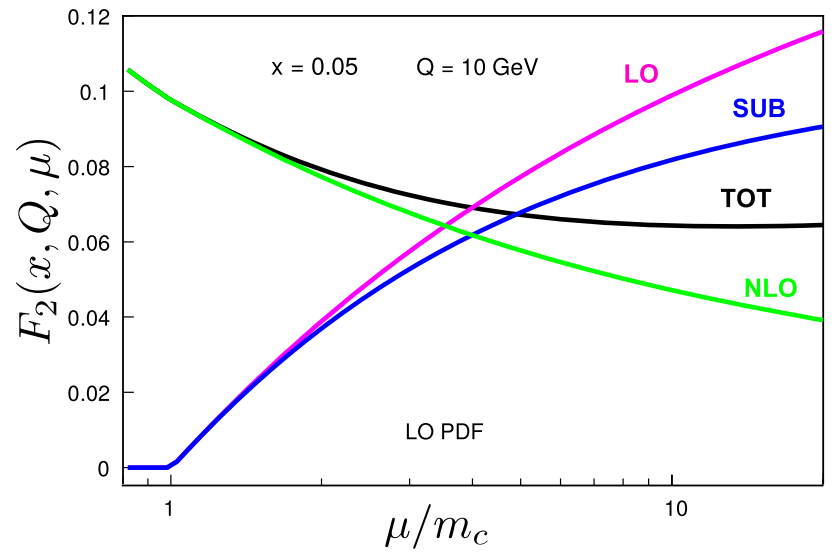

Fig. 18 Calculation of $F_{2}^{c}$ vs. $\mu$ in the VFNS illustrating the cancellation of the $\operatorname{LO}\left(\bar{c} W^{+} \rightarrow \bar{s}\right)$ and the SUB $(g \rightarrow \bar{c}) \otimes\left(\bar{c} W^{+} \rightarrow \bar{s}\right)$ contributions in the region $\mu \sim m_{c}$. The $Q$ scale is fixed at $10 \mathrm{GeV}$ and the charm PDF is matched at $\mu_{c}=m_{c}$ such that $f_{c}\left(x, \mu=m_{c}\right)=0$

Explanation \#1: matching of LO and SUB: Recall that in the $t$-channel case, the subtraction term SUB removed the double counting between the LO $s W^{+} \rightarrow c$ and NLO $g W^{+} \rightarrow c \bar{s}$ subprocesses.

The $u$-channel case is analogous in that this subtraction term removes the double counting between the $\mathrm{LO} \bar{c} W^{+} \rightarrow \bar{s}$ and NLO $g W^{+} \rightarrow c \bar{s}$ subprocesses; both contributions are required to ensure that the resulting cross section is insensitive to the scale $\mu$.

This is apparent in Fig. 18 where we plot the individual terms versus $\mu$ for fixed values of $x_{\mathrm{Bj}}$ and $Q$. In the region $\mu \sim m_{c}$, the charm PDF $f_{c}(x, \mu)$ (and hence, the LO contribution) rises very quickly as the DGLAP evolution is driven by the very large gluon distribution via $g \rightarrow c \bar{c}$ splitting, and combined with a large $\alpha_{s}(\mu)$. The SUB subtraction also rises quickly as this is driven by the logarithmic term $\ln \left(\mu^{2} / m_{c}^{2}\right)$. The difference $(L O-S U B)$ is the physical contribution to the total $[T O T=L O+N L O-S U B]$, and it is this combination that is smooth across the "turn on" of the charm PDF at the matching scale $\mu_{c}=m_{c}$. We now see that if we neglect the LO $\left(\bar{c} W^{+} \rightarrow \bar{s}\right)$ contribution, we lose the cancellation between LO and SUB in the region $\mu \sim m_{c}$, and our structure function (or cross section) would have an anomalous shift at the arbitrarily location $\left(\mu_{c}\right)$ where we turn on the charm PDF.

As we vary the unphysical scale $\mu$, we are simply shifting contributions between the separate $\{L O, N L O, S U B\}$ terms which individually exhibit a large $\mu$-dependence. However, the total combination ( $T O T$ ), which represents the physical observable, is relatively insensitive to $\mu$ (up to higher orders), and this property is evident in Fig. 18.

Explanation \#2: removing “double counting:" A second way to understand why we require the LO process $\bar{c} W^{+} \rightarrow \bar{s}$ is to consider the regions of phase space covered by each of the subprocesses. The singularity of the $u$-channel NLO $g W^{+} \rightarrow c \bar{s}$ processes arises from the phase-space region where the intermediate $\bar{c}$-quark becomes collinear and close to the mass shell. ${ }^{13}$ This is precisely the phase-space region of the LO process $\bar{c} W^{+} \rightarrow \bar{s}$ where the partonic $\bar{c}$-quark is collinear to the hadron. The SUB term then removes the "double counting" between the LO and NLO contributions; hence, all three contributions $\{L O, N L O, S U B\}$ are necessary to cover the full phase space.

This is also apparent if we consider the transverse momentum $\left(p_{T}\right)$ of the final-state charm in the Breit frame. For the LO $\bar{c} W^{+} \rightarrow \bar{s}$ process in the Breit frame, the incoming $W^{+}$ and $\bar{c}$ are collinear, and the produced $\bar{s}$ must have zero $p_{T}$ in this frame.

For the NLO $g W^{+} \rightarrow c \bar{s}$ process, we integrate over the complete phase space for the exchanged $\bar{c}$ quark, and this will include the region where the $\bar{c}$-quark is emitted nearly collinear to the gluon and nearly on-shell; in this region the $\bar{c}$-quark will have $p_{T} \sim 0$ and we encounter a singularity from the internal $\bar{c}$-quark propagator. The $p_{T} \sim 0$ region is precisely that subtracted by the SUB counter term ${ }^{14}$ and this ensures that the combination $(N L O-S U B)$ is free of divergences.

Recap: To recap, i) the combination of the LO and SUB terms ensure a minimal $\mu$ variation at low $\mu$, and ii) the combination of SUB and NLO ensures that the mass singularities are cancelled at high $\mu$.

This interplay of terms illustrates some of the intricacies of QCD, especially since this exchange is across different orders of $\alpha_{s}$.

Furthermore, note that in the $u$-channel for both the LO and SUB contributions, the charm quark is collinear to the incoming hadron, and thus exits in the hadron remnants. While this may be experimentally difficult to observe, because we are asking for a "fully inclusive" $F_{2}^{c}$, these contributions cannot be simply ignored. We will discuss this further in the following section.

Defining $F_{2}^{c}$ : The LO $u$-channel $\bar{c} W^{+} \rightarrow \bar{s}$ process foreshadows difficulties that we encounter if we try and extend the concept of "fully inclusive" $F_{2}^{c}$ to higher orders. We note that in Ref. [63] Collins extended the proof of factorization to include heavy quarks such as charm and bottom for an inclusive structure function $F_{2}$; analysis of a "fully inclusive" $F_{2}^{c}$ is more complex for a number of reasons. Whereas $F_{2}$ only requires measurement of the outgoing lepton energy and angle, $F_{2}^{c}$ also requires information on the hadronic final state. At the parton level, this introduces complications

\footnotetext{
$\overline{13}$ For example, the $c$-quark is off-shell by the order of its mass $m_{c}$; this is independent of the scale $Q$ and does not assume any $Q \gg m_{c}$ limit.

14 Specifically, the incoming $W^{+}$and $g$ are collinear and the gluon then emits a collinear $c \bar{c}$ pair so the final $\bar{s}$ has zero $p_{T}$.
} 


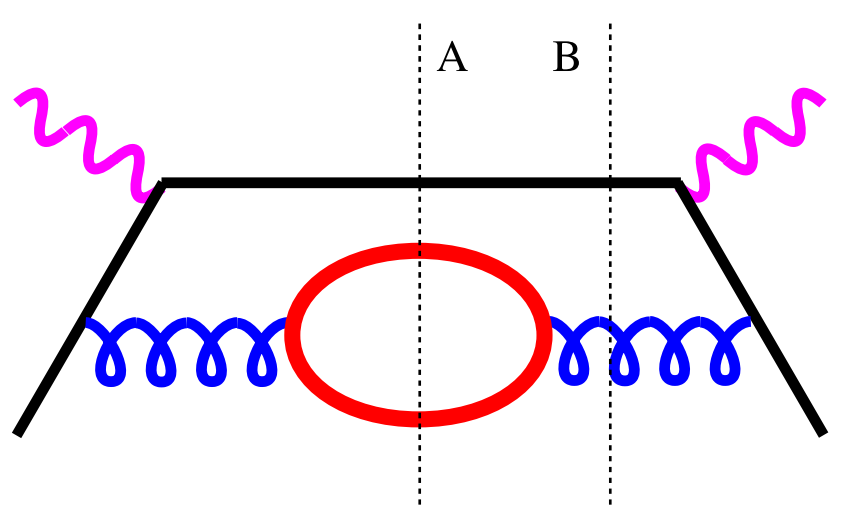

Fig. 19 A higher order Feynman graph illustrating the complications in defining a "fully inclusive" $F_{2}^{\text {charm }}$. A light quark $(q)$ scatters from a vector boson $(V)$ with a $c \bar{c}$ in the internal loop. If we cut the amplitude at "A" we have charm in the final state and this must be included in $F_{2}^{\text {charm }}$. If we cut the amplitude with cut " $\mathrm{B}$ " there is no charm in the final state. Additionally, since this diagram contributes to the beta function, this highlights the complications of using an $\alpha_{S}$ and hard scattering $\hat{\sigma}$ with differing $N_{\text {eff }}$

including when the charm is in the hadronic remnants and brings in both fragmentation and fracture functions.

To characterize the theoretical issues involved in constructing $F_{2}^{c}$, we can imagine starting from the (well-defined) inclusive $F_{2}$, and then dividing the contributions into two sets: one for $F_{2}^{c}$ for the "heavy" charm quark, and the rest into $F_{2}^{u, d, s}$ for the "light" quarks. We will show that this theoretical procedure encounters ambiguities.

The LO $u$-channel $\bar{c} W^{+} \rightarrow \bar{s}$ process does not have any "apparent" charm quark in the final state, but this contribution is essential to balance with the SUB process $f_{g} \otimes \widetilde{f}_{g \rightarrow \bar{c}} \otimes$ $\sigma_{\bar{c} W^{+} \rightarrow \bar{s}}$. Note that for the SUB process the charm quark arises from a gluon splitting into a collinear $c \bar{c}$ pair which is then part of the hadron remnants. For the LO process, presumably our $\bar{c}$ quark also came from a gluon splitting into a collinear $c \bar{c}$ pair. Thus, our $F_{2}^{c}$ must include those cases where the charm is contained in the hadron remnants.

This issues touches on the fact that, because the charm parton ultimately fragments into a charmed hadron (typically a $D$ meson), we must introduce a set of fragmentation functions (FFs) which are scale-dependent and will factorize final-state singularities in a similar manner as the PDFs factor the initial-state singularities. ${ }^{15}$ Specifically, we may also allow for the possibility that a gluon or a light quark fragments into a charmed hadron.

The bubble diagram: Some of the theoretical intricacies of defining a "fully inclusive" $F_{2}^{c}$ are illustrated in Fig. 19 which shows a higher-order DIS process with a quark-antiquark loop.

\footnotetext{
$\overline{15}$ For the NLO quark-initiated contributions (not shown) we will have final state singularities from processes such as $c \rightarrow c g$ which will be factorized into the FFs.
}

Let us compute this diagram in the $n_{f}=3$ FFNS where the internal loop is a massive $c \bar{c}$-pair and the external quark is a light quark $\{u, d, s\}$. If the final state is represented by Cut-A, then we have charm quarks in the final state, and this should be included in $F_{2}^{c}$.

However, if we instead use Cut-B as a final state, there is no charm in the final state, so this should not be included in $F_{2}^{c}$. [More precisely, when we renormalize the charm loop with zero-momentum subtraction, this contribution effectively decouples.] Thus, the contribution from Cut-A will be included in $F_{2}^{c}$, but the contribution from Cut-B will not.

This diagram generates additional complications in that multiple quark flavors are involved. For example, the bubble diagram involves quarks of both $q=\{u, d, s\}$ and $c$ flavors, so this contribution cannot be uniquely assigned to $F_{2}^{q}$ or $F_{2}^{c}$. We can introduce theoretical definitions to make the choice, but then we have to be careful about double-counting contributions and introducing uncancelled singularities. For example, the bubble diagram of Fig. 19 is encountered in the $F_{2}^{c}$ heavy-quark calculations of Refs. [64,65]; here, an additional scale $\Delta$ is introduced to subdivide the contributions.

The running of $\alpha_{s}$ in the FFNS: The bubble diagram of Fig. 19 also highlights the difficulty of using a $n_{f}=3$ FFNS with a VFNS running of $\alpha_{s}$. In a $n_{f}=3$ FFNS, internal $c \bar{c}$ loops decouple from the theory and are not included in the calculation; ${ }^{16}$ however, the $\beta$-function with $n_{f}=4$ requires precisely these $c \bar{c}$ loop contributions. This deficiency can be patched order by order by expanding the $\beta$-function and inserting the required terms at each order [66-68]. Once again, we cannot unambiguously divide the inclusive $F_{2}$ into separate "light" and "heavy" quantities.

Extensions to bottom and top: While we have used the charm quark to illustrate these features, the same properties can, in principle, be applied to both the bottom and top quark. ${ }^{17}$ For the case of the bottom quark, the larger mass $m_{b}$ yields a smaller $\alpha_{s}(\mu)$ for $\mu \sim m_{b}$ and the evolution of $f_{b}(x, \mu)$ is thus reduced. Nevertheless, for large-scale processes (such as at the LHC) we often find it convenient to make use of $f_{b}(x, \mu)$ and treat the bottom on an equal footing as the other light quarks. For the case of the top quark, the very large mass $m_{t}$ yields a much smaller $\alpha_{s}(\mu)$ for $\mu \sim m_{t}$ and the evolution of $f_{t}(x, \mu)$ is comparatively reduced.

\footnotetext{
16 More precisely, the heavy quarks are renormalized with zeromomentum subtraction and their contributions decouple; this is why we can neglect loops from the the top quark and any other heavy particle.

17 Additionally, Collins definitively addressed the case of multiple heavy quarks which can allow for both charm and bottom in a unified framework; in contrast to some incorrect claims in the literature, there is no difficulty in including multiple heavy quarks. (cf. Ref. [63], Sec. IX.)
} 
Summary To properly define $F_{2}^{c}$ at higher orders, we encounter the theoretical issues discussed above: as the charm quark fragments into a charmed meson, we must be careful to ensure that the theoretical quantity matches what is actually measured experimentally. This is more complex than simply asking for the portion of $F_{2}$ has a charm in the final state, and is an issue for both the FFNS and VFNS as we move to higher orders. We can perform the computation in the FFNS but in the large energy limit we encounter $\ln \left(Q^{2} / m_{c}^{2}\right)$ divergences and this, in part, contributes to the observed differences at large $Q$.

The VFNS includes the charm quark as an active parton for $\mu$ scales above a matching scale $\mu_{c}$. For large $Q$ scales, the mass singularities of NLO and SUB terms will cancel to yield a result free of divergences. For scales $\mu \sim m_{c}$, cancellation between the LO and SUB contributions ensures a minimal $\mu$ dependence; however, as this can be delicate to implement numerically, we have the option of displacing the matching scale $\mu_{c}$ to a larger scale where the cancellation is more stable [51,61].

\section{References}

1. H. Abdolmaleki et al., Impact of low-x resummation on QCD analysis of HERA data. Eur. Phys. J. C 78(8), 621 (2018). arXiv: 1802.00064 [hep-ph]

2. H. Abramowicz et al., Combination of measurements of inclusive deep inelastic $e^{ \pm} p$ scattering cross sections and QCD analysis of HERA data. Eur. Phys. J. C 75(12), 580 (2015). arXiv:1506.06042 [hep-ex]

3. J. Gao, L. Harland-Lang, J. Rojo, The structure of the proton in the LHC precision. Era. Phys. Rept. 742, 1-121 (2018). arXiv:1709.04922 [hep-ph]

4. S. Alekhin et al., Parton distribution functions, as, and heavyquark masses for LHC Run II. Phys. Rev. D 96(1), 014011 (2017). arXiv: 1701.05838 [hep-ph]

5. R.D. Ball et al., Parton distributions from highprecision collider data. Eur. Phys. J. C 77(10), 663 (2017). arXiv:1706.00428 [hep$\mathrm{ph}]$

6. O. Behnke, A. Geiser, M. Lisovyi, Charm, beauty and top at HERA. Prog. Part. Nucl. Phys. 84, 1-72 (2015). arXiv:1506.07519 [hepex]

7. O. Zenaiev, Charm production and QCD analysis at HERA and LHC. Eur. Phys. J. C 77(3), 151 (2017). arXiv:1612.02371 [hepex]

8. H. Abdolmaleki, A. Khorramian, A. Aleedaneshvar, Impact of intrinsic charm on PDFs with EMC and LHC data. Nucl. Part. Phys. Proc. 282-284, 27-31 (2017)

9. H. Abdolmaleki, A. Khorramian, Parton distribution functions and constraints on the intrinsic charm content of the proton using the Brodsky-Hoyer-Peterson-Saka approach. Phys. Rev. D 99(11), 116019 (2019). arXiv:1903.02583 [hep-ph]

10. E. Laenen et al., Complete O (alpha-s) corrections to heavy flavor structure functions in electroproduction. Nucl. Phys. B 392, 162 228 (1993)

11. E. Laenen et al., O(alpha-s) corrections to heavy flavor inclusive distributions in electroproduction. Nucl. Phys. B 392, 229-250 (1993)
12. T. Gottschalk, Chromodynamic corrections to neutrino production of heavy quarks. Phys. Rev. D 23, 56 (1981)

13. M. Gluck, S. Kretzer, E. Reya, Detailed next-to-leading order analysis of deep inelastic neutrino induced charm production off strange sea partons. In: Phys. Lett. B 398 (1997). [Erratum: Phys. Lett.B405,392(1997)], pp. 381-386. arXiv:hep-ph/9701364 [hep$\mathrm{ph}]$

14. J. Blumlein et al., $O\left(\alpha_{s}\right)$ heavy flavor corrections to charged current deep-inelastic scattering in mellin space. Phys. Lett. B 700, 294 304 (2011). arXiv:1104.3449 [hep-ph]

15. M. Buza, W.L. van Neerven, $\mathrm{O}$ (alpha-s**2) contributions to charm production in charged current deep inelastic lepton - hadron scattering. Nucl. Phys. B 500, 301-324 (1997). arXiv:hep-ph/9702242 [hep-ph]

16. J. B1ÂOEmlein, A. Hasselhuhn, T. Pfoh, The $O\left(\alpha_{s}^{2}\right)$ heavy quark corrections to charged current deep-inelastic scattering at large virtualities. Nucl. Phys. B 881, 1-41 (2014). arXiv:1401.4352 [hep$\mathrm{ph}]$

17. E.L. Berger et al., Charm-Quark production in deep-inelastic neutrino scattering at nextto-next-to-leading order in QCD. Phys. Rev. Lett. 116(21), 212002 (2016). arXiv:1601.05430 [hep-ph]

18. A. Behring et al., $O\left(\alpha_{s}^{3}\right)$ heavy flavor contributions to the charged current structure function $x F_{3}\left(x, Q^{2}\right)$ at large momentum transfer. Phys. Rev. D 92(11), 114005 (2015). arXiv:1508.01449 [hep-ph]

19. T. Aaltonen et al., First measurement of the production of a $W$ boson in association with a single charm quark in $p \bar{p}$ collisions at $\sqrt{s}=$ 1.96 - TeV. Phys. Rev. Lett. 100, 091803 (2008). arXiv:0711.2901 [hep-ex]

20. V.M. Abazov et al., Measurement of the ratio of the $p \bar{p} \rightarrow W^{+} c^{-}$ jet cross section to the inclusive $p \bar{p} \rightarrow W+$ jets cross section. Phys. Lett. B 666, 23-30 (2008). arXiv:0803.2259 [hep-ex]

21. V.M. Abazov et al., Measurement of the $W+b$-jet and $W+c$ jet differential production cross sections in $p \bar{p}$ collisions at $\sqrt{s}=$ 1.96TeV. Phys. Lett. B 743, 6-14 (2015). arXiv:1412.5315 [hepex]

22. S. Chatrchyan et al., Measurement of associated $\mathrm{W}+$ charm production in pp collisions at $\sqrt{s}=7 \mathrm{TeV}$. JHEP 02, 013 (2014). arXiv: 1310.1138 [hep-ex]

23. G. Aad et al., Measurement of the production of a $W$ boson in association with a charm quark in pp collisions at $\sqrt{s}=7 \mathrm{TeV}$ with the ATLAS detector. JHEP 05, 068 (2014). arXiv:1402.6263 [hep-ex]

24. A.M. Sirunyan et al., Measurement of associated production of a $\mathrm{W}$ boson and a charm quark in protonproton collisions at $\sqrt{s}=$ 13TeV. Eur. Phys. J. C 79(3), 269 (2019). arXiv:1811.10021 [hepex]

25. R. A. Khalek et al., Probing proton structure at the large hadron electron collider. (2019). arXiv:1906.10127 [hep-ph]

26. G. Kramer, B. Lampe, H. Spiesberger, Scheme and scale dependence of charm production in neutrino scattering. Z. Phys. C 72, 99-106 (1996). arXiv:hep-ph/9511396 [hep-ph]

27. W.G. Seligman et al., Improved determination of alpha(s) from neutrino nucleon scattering. Phys. Rev. Lett. 79, 1213-1216(1997). arXiv:hep-ex/9701017 [hep-ex]

28. M. Tzanov et al., Precise measurement of neutrino and antineutrino differential cross sections. Phys. Rev. D 74, 012008 (2006). arXiv:hep-ex/0509010 [hep-ex]

29. G. Onengut et al., Measurement of nucleon structure functions in neutrino scattering. Phys. Lett. B 632, 65-75 (2006)

30. J.P. Berge et al., A measurement of differential cross-sections and nucleon structure functions in charged current neutrino interactions on iron. Z. Phys. C 49, 187-224 (1991)

31. O. Samoylov et al., A precision measurement of charm dimuon production in neutrino interactions from the NOMAD experiment. Nucl. Phys. B 876, 339-375 (2013). arXiv:1308.4750 [hep-ex] 
32. A. Airapetian et al., Measurement of parton distributions of strange quarks in the nucleon from charged-kaon production in deepinelastic scattering on the deuteron. Phys. Lett. B 666, 446-450 (2008). arXiv:0803.2993 [hep-ex]

33. I. Abt et al., Charm production in charged current deep inelastic scattering at HERA. JHEP 05, 201 (2019). arXiv:1904.03261 [hepex]

34. H.L. Lai et al., The Strange parton distribution of the nucleon: global analysis and applications. JHEP 04, 089 (2007). arXiv:hep-ph/0702268 [hep-ph]

35. T. Aaltonen et al., Observation of the production of a W Boson in association with a single charm quark. Phys. Rev. Lett. 110(7), 071801 (2013). arXiv:1209.1921 [hep-ex]

36. T.A. Aaltonen et al., Measurement of vector boson plus $D *(2010)^{+}$ meson production in $\bar{p} p$ collisions at $\sqrt{s}=1.96 \mathrm{TeV}$. Phys. Rev. D 93.5, 052012 (2016). arXiv:1508.06980 [hep-ex]

37. S. Kretzer et al., Cteq6 parton distributions with heavy quark mass effects. Phys. Rev. D 69, 114005 (2004). arXiv:hep-ph/0307022 [hep-ph]

38. A.D. Martin et al., Physical gluons and high E $(T)$ jets. Phys. Lett. B 604, 61-68 (2004). arXiv:hep-ph/0410230 [hep-ph]

39. M. Aaboud et al., Precision measurement and interpretation of inclusive $W^{+}, W^{-}$and $Z / \gamma *$ production cross sections with the ATLAS detector. Eur. Phys. J. C 77(6), 367 (2017). arXiv:1612.03016 [hep-ex]

40. A.M. Cooper-Sarkar, K. Wichmann, QCD analysis of the ATLAS and $\mathrm{CMS} W^{ \pm}$and $\mathrm{Z}$ cross-section measurements and implications for the strange sea density. Phys. Rev. D 98(1), 014027 (2018). arXiv: 1803.00968 [hep-ex]

41. S. Alekhin et al., HERAFitter. Eur. Phys. J. C 75(7), 304 (2015). arXiv: 1410.4412 [hep-ph]

42. J.L. Abelleira Fernandez et al., A large hadron electron collider at CERN: report on the physics and design concepts for machine and detector. J. Phys. G 39, 075001 (2012). arXiv:1206.2913 [physics.acc-ph]

43. M. Tanabashi et al., Review of particle physics. Phys. Rev. D 98(3), 030001 (2018)

44. S. Alekhin, J. Blumlein, S. Moch, Parton distribution functions and benchmark cross sections at NNLO. Phys. Rev. D 86, 054009 (2012). arXiv:1202.2281 [hep-ph]

45. S. Alekhin et al., Determination of strange sea quark distributions from fixed-target and collider data. Phys. Rev. D 91(9), 094002 (2015). arXiv:1404.6469 [hep-ph]

46. S. Alekhin, J. BlÃOEmlein, S. Moch, NLO PDFs from the ABMP16 fit. Eur. Phys. J. C 78(6), 477 (2018). arXiv:1803.07537 [hep-ph]

47. S. Alekhin, OPENQCDRAD. http://wwwzeuthen.desy.de/ alekhin/OPENQCDRAD/

48. S. Forte et al., Heavy quarks in deep-inelastic scattering. Nucl. Phys. B 834, 116-162 (2010). arXiv:1001.2312 [hep-ph]

49. V. Bertone, S. Carrazza, J. Rojo, APFEL: a PDF evolution library with QED corrections. Comput. Phys. Commun. 185, 1647-1668 (2014). arXiv:1310.1394 [hep-ph]

50. A. Buckley et al., LHAPDF6: parton density access in the LHC precision era. Eur. Phys. J. C 75, 132 (2015). arXiv:1412.7420 [hep-ph]
51. V. Bertone et al., Impact of the heavy quark matching scales in PDF fits. Eur. Phys. J. C 77(12), 837 (2017). arXiv: 1707.05343 [hep-ph]

52. A. Kusina et al., Hybrid scheme for heavy flavors: merging the fixed flavor number scheme and variable flavor number scheme. Phys. Rev. D 88(7), 074032 (2013). arXiv:1306.6553 [hep-ph]

53. J. Gao, Massive charged-current coefficient functions in deepinelastic scattering at NNLO and impact on strange-quark distributions. JHEP 02, 026 (2018). arXiv: 1710.04258 [hep-ph]

54. M.A.G. Aivazis et al., Leptoproduction of heavy quarks. 2. A Unified QCD formulation of charged and neutral current processes from fixed target to collider energies. Phys. Rev. D 50, 3102-3118 (1994). arXiv:hep-ph/9312319 [hep-ph]

55. H. Paukkunen, P. Zurita, PDF reweighting in the Hessian matrix approach. JHEP 12, 100 (2014). arXiv:1402.6623 [hep-ph]

56. J. Blumlein, M. Klein, On the cross calibration of calorimeters at e p colliders. Nucl. Instrum. Meth. A 329, 112-116 (1993)

57. ATLAS and CMS Collaborations. Report on the Physics at the HLLHC and Perspectives for the HELHC. In: HL/HE-LHC Physics Workshop: final jam-boree Geneva, CERN, March 1, 2019. 2019. arXiv:1902.10229 [hep-ex]

58. J. L. Abelleira Fernandez, et al., On the Relation of the $\mathrm{LHeC}$ and the LHC. (2012). arXiv:1211.5102 [hep-ex]

59. M. Klein, Future Deep Inelastic Scattering with the LHeC. In: From My Vast Repertoire... Guido Altarelli's Legacy. Ed. by Aharon Levy, Stefano Forte and Giovanni Ridolfi. 2019, pp. 303-347. arXiv:1802.04317 [hep-ph]

60. T. Stavreva et al., Heavy quark production in the ACOT scheme at NNLO and N3LO. Phys. Rev. D 85, 114014 (2012). arXiv:1203.0282 [hep-ph]

61. V. Bertone, et al., xFitter 2.0.0: Heavy quark matching scales: Unifying the FFNS and VFNS. PoS DIS2018 (2018), p. 015. 1808.08623 [hep-ph]

62. J.C. Collins, F. Wilczek, A. Zee, Low-energy manifestations of heavy particles: application to the neutral current. Phys. Rev. D 18, 242 (1978)

63. J.C. Collins, Hard scattering factorization with heavy quarks: a general treatment. Phys. Rev. D 58, 094002 (1998). arXiv:hep-ph/9806259 [hep-ph]

64. A. Chuvakin, J. Smith, W.L. van Neerven, Comparison between variable flavor number schemes for charm quark electroproduction. Phys. Rev. D 61, 096004 (2000). arXiv:hep-ph/9910250 [hep-ph]

65. A. Chuvakin, J. Smith, W.L. van Neerven, Bottom quark electroproduction in variable flavor number schemes. Phys. Rev. D 62, 036004 (2000). arXiv:hep-ph/0002011 [hep-ph]

66. D. Napoletano. Thesis: a new hybrid scheme for the treatment of heavy quarks in perturbative QCD. In: Istituto Nazionale di Fisica Nucleare I Sezione di Milano (2014)

67. I. Bierenbaum, J. Blumlein, S. Klein, The gluonic operator matrix elements at $\mathrm{O}(\mathrm{alpha}(\mathrm{s}) * * 2)$ for DIS heavy flavor production. Phys. Lett. B 672, 401-406 (2009). arXiv:0901.0669 [hep-ph]

68. F. Cascioli et al., NLO matching for $t \bar{t} b \bar{b}$ production with massive b-quarks. Phys. Lett. B 734, 210-214 (2014). arXiv:1309.5912 [hep-ph] 